\title{
PICASSO, LAS CABEZAS MODELADAS DE MARÍA TERESA WALTER EN \\ BOISGELOUP EN 1930-31 Y 1934
}

\author{
ANDRÉS LUQUE TERUEL \\ UNIVERSIDAD DE SEVILLA
}

\begin{abstract}
RESUMEN: La nueva escultura modelada de Picasso en Boisgeloup muestra un sólido dominio estructural y el conocimiento de la estatuaria clásica como punto de partida para la deformación orgánica que lo llevó a una nueva renovación formal. El paso gradual hacia las abstracciones orgánicas y la definición de una configuración estructural propia, conocida como nariz-frente, quedaron definidas en la serie formada por distintos estados titulados Cabeza de María Teresa Walter, algunos expuestos en el Pabellón de España en la Exposición Universal de París, en el año 1937.
\end{abstract}

PALABRAS ClAVE: Picasso, Escultura, Vanguardias, Abstracción Orgánica, Informalismo

\section{PICASSO, THE MODELED HEADS OF MARIA TERESA WALTER IN BOISGELOUP, 1930-31 AND 1934. A NECESSARY REVIEW AS SERIES}

\begin{abstract}
The new Picasso's sculpture shape in Boisgeloup sign a solid structural dominion and classical statuary knowledge as a departure point for organic deformation leading to a new formal renovation. The gradual move toward organic abstraction and the definition of a proper structural shape, known as nose-brow, were defined in some states of the Maria Teresa Walter's head, expose in the Hispanic Pavilion of Paris Universal Exposition of 1937.
\end{abstract}

KEYWORDS: Picasso, Sculpture, Avant-Garde, Organic Abstraction, No formalist Recibido: 01/07/2015/Aceptado: 07/06/2016 
Después de dos décadas en las que inventó procedimientos y recursos con los que operó desde perspectivas creativas inéditas, con las que cambió la orientación de la escultura de vanguardia rompiendo cualquier tipo de límite, Picasso retomó los procedimientos de la escultura modelada en el taller rural de Boisgeloup, en 1930, y lo hizo determinando novedades formales no menos sorprendentes y avanzadas. A diferencia de las esculturas que modeló en París en 1928 y 1929, con las que anticipó el sentido de la evolución, en concreto, Bañista (Metamorfosis) I y II, y Mujer sentada I y II, excepciones en pequeño formato y modeladas en barro que forman parte de una producción amplia en la que predominaron los dibujos escultóricos, en este taller modeló numerosas esculturas, la mayoría en yeso y en tamaños muy diversos.

Distintas aplicaciones de su sistema creativo, basado en la intuición plástica en estados consecutivos derivados de las cualidades originales de la forma utilizada como referencia inicial, y la posterior selección mediante un proceso de cálculo, por lo tanto intelectivo a la vez, coinciden en la referencia natural sobre la que experimentó deformaciones biomórficas, en las que impuso la naturaleza orgánica de los elementos y las configuraciones. Muchas de esas aplicaciones están bien identificadas y de ellas se conservan secuencias amplias y completas; una de las más significativas es la titulada Cabeza de María Teresa Walter, como tal, formada por una serie de bustos en estados consecutivos y directamente relacionados; otra, con el mismo tema y título, resuelta con modelados en relieve $^{1}$. Esto induce a la revisión necesaria como unidades relacionadas entre sí en el contexto de una misma serie.

Cuatro esculturas de esa serie estuvieron expuestas en el Pabellón de España de la Exposición Internacional de París del año 1937, diseñado por el arquitecto José Luis Sert. Celebrada en plena Guerra Civil española, fue muy bien estudiado por Fernando Martín Martín ${ }^{2}$. El estado de Francia invitó al gobierno republicano como legítimo de España, y obvió a los militares sublevados, y, como expusieron Fernando Martín Martín y después Herschel B. Chipp, el espacio racionalista diáfano estuvo presidido por Guernica ${ }^{3}$, la pintura con la que el propio Picasso lamentó el bombardeo de dicha localidad y, por extensión, el sufrimiento del pueblo español. Frente a ella podía verse la fuente de Alexander Calder, titulada Mercurio español de Almadén ${ }^{4}$; en el testero opuesto una fotografía de gran tamaño del

\footnotetext{
${ }^{1}$ AAVV (Comisarios: John Richardson y Diana Widmaier): L'amour fou. Picasso and Maria-Thérèse, Nueva York, Galerie Gagosian, 2011. AAVV: Picasso Sculpture, Nueva York, Museo de Arte Moderno de Nueva York.

2 MARTÍN MARTÍN, Fernando: El Pabellón Español en la Exposición Universal de París en 1937, Sevilla, Universidad de Sevilla, 1982.

${ }^{3}$ CHIPP, Herschel B.: Picasso's Guernica. History, Transformations, Meanings, Berkeley y Los Ángeles, University of California Press, 1988. Barcelona, Polígrafa, 1991, pp. 145-149.

${ }^{4}$ BAAL-TESHUVA, Jacob: Alexander Calder, Colonia, Taschen, 1998, p. 23.
} 
poeta Federico García Lorca, asesinado meses antes por las fuerzas nacionalistas; y, junto a la escalera, un mural con alusiones a la guerra civil española de Joan Miró, El segador, con más de cinco metros de altura. En la planta superior, aparecía un poema de Paul Éluard, titulado, La victoire de Guernica, junto a una fotografía de la propia ciudad vasca bombardeada. Frente a la entrada, en el auditorio al aire libre, se proyectaban continuamente documentales sobre la guerra bajo la dirección de Luis Buñuel. Fuera, junto a la puerta, la escultura monumental y abstracta de Alberto Sánchez, El pueblo español sigue un camino que conduce a una estrella ${ }^{5}$, constituía el auténtico símbolo de la firmeza y la grandeza democrática de ese pueblo; representado de modo específico por el grito desgarrado de otra escultura próxima, La Montserrat ${ }^{6}$, obra figurativa forjada en hierro por Julio González. Las réplicas vaciadas en cemento de las cuatro cabezas de María Teresa Walter modeladas unos años antes por Picasso completaban los espacios exteriores del perímetro del edificio.

\section{ESTADO DE LA CUESTIÓN SOBRE UNA APLICACIÓN COMPLETA, LAS CABEZAS DE MARÍA TERESA WALTER (1930 A 1931)}

El Busto de mujer que Picasso talló en madera en 1930 es una obra modesta en cuanto a su formato mas de una importancia hasta ahora no señalada como estado plástico previo a la evolución de la serie de variaciones a la que pertenecen Cabeza de María Teresa Walter I, Cabeza en relieve de María Teresa Walter I, Busto de María Teresa Walter, Cabeza de María Teresa Walter II, Cabeza en relieve de María Teresa Walter II, Cabeza de María Teresa Walter III, todas en 1931; y los relieves Posible Cabeza de María Teresa Walter I a IX, y, como último estado, Dama Oferente, éstas en 1933.

De las esculturas citadas, las cuatro que estuvieron en el Pabellón de España de la Exposición Universal de París, en 1937, son: Cabeza de María Teresa Walter II, que figuró como Cabeza de mujer, Busto de Maria Teresa Walter, con el título de Gran cabeza de mujer, Cabeza de María Teresa Walter III, tal Cabeza de mujer, y Dama Oferente ${ }^{7}$. Para ello, Picasso realizó vaciados en cemento ${ }^{8}$, cuya presencia en el pabellón estudió Fernando Martín Martín con indudable éxito?. Según Josefina Alix, allí estuvo también una réplica en bronce de la

\footnotetext{
${ }^{5}$ MARTIN, Peter, Alberto, Budapest, Éditions Corvina, 1964, pp. 20 y 21.

${ }^{6}$ GUIGON, Enmanuel, Julio González. Esculturas y dibujos en la colección del IV AM, Sevilla, Caja San Fernando, 2000, pp. 15-17.

${ }^{7}$ VAN HENSBERGEN, Gijs, Guernica. La historia de un icono del siglo XX, Madrid, Editorial Debate, 2005.

${ }^{8}$ El de Busto de María Teresa Walter en el Museo Picasso, París. Los de Cabeza de María Teresa Walter II y III en el Museo de Antibes. El de Dama Oferente, perdido.

${ }^{9}$ MARTÍN MARTÍN, Fernando: op. cit., pp. 92 a 96.
} 
escultura de Picasso Bañista de Boisgeloup ${ }^{10}$, del año 1931, pero ésta, aunque es de la época, forma parte de otra aplicación distinta del sistema de Picasso.

Werner Spies reconoció la unidad de las cuatro cabezas y el primero de los relieves $^{11}$, y los consideró obras de un mismo grupo en fases sucesivas que superan el clasicismo de la primera y derivan de los bustos, ya lineales, ya biomórficos, insertos en un tema frecuente de representación, el taller del escultor, protagonista en varias pinturas independientes, como la titulada, El escultor ${ }^{12}$, en París, en 1931, y un grupo de grabados de la Suite Vollard $^{13}$. Con ello, tuvo en cuenta la capacidad de transformación y los distintos criterios de Picasso en Boisgeloup. Su mérito, indiscutible, fue que apreció la realidad y las relaciones de la serie, derivadas de una aplicación concreta del sistema creativo, de índole plástica y progresivo en cuanto a los hallazgos derivados de la creatividad técnica y formal sobre las cualidades innatas de las referencias, aunque no lo reconociese como tal, al menos de modo explícito.

Para su interpretación partió del testimonio de Picasso a Tériade ${ }^{14}$ :

"Si se parte de la imagen naturalista y se quiere llegar por eliminaciones sucesivas a la forma pura, el volumen nítido sin nada accidental, uno termina necesariamente en el huevo. De forma análoga, partiendo del huevo y siguiendo el mismo camino en sentido inverso se llega al retrato, que es la meta opuesta".

Werner Spies propuso un elemento clave en la evolución y en la interpretación de estas obras, la estructura denominada nariz-frente, que es la integración de ambos elementos en una sola protuberancia. Detectó esta cualidad formal en los proyectos de Las Señoritas de Aviñón, en 1906 y 1907, según él influidos por una Máscara pectoral Nimba, cuestión que rechazó Picasso y quedó descartada. Citó además antecedentes fálicos del arte etnológico, con especial interés en la nariz-falo de una Máscara japonesa Gigaku, en el siglo VIII, reproducida en los Cahiers d'Art ${ }^{15}$.

La capacidad de Werner Spies es admirable. Comparó las deformaciones y la belleza no ortodoxa de Picasso, con la representación excéntrica del movimiento en Louis Aragon y André Bretón ${ }^{16}$, y la atracción del feísmo con los postulados de Bataille; mas

\footnotetext{
${ }^{10}$ ALIX TRUEBA, Josefina, Pabellón Español en la Exposición Universal de París en 1937, Madrid, Centro de Arte Reina Sofía, 1987, p. 235.

11 SPIES, Werner: La escultura de Picasso, Barcelona, Polígrafa, 1989. pp. 149-158.

${ }^{12}$ Museo Picasso, París. Óleo sobre contrachapado, 128’5 x 96 cm. Zervos (Z) VII, 346; Museo Picasso de París (MPP) 135.

13 SPIES, Werner: op. cit., pp. 149 y 150.

14 TÉRIADE, E.: "Conversation avec Picasso"; en L'Intransigeant, París, 16 de junio de 1932.

15 Cabiers d'Art, París, p. 376.

16 ARAGON, Louis y BRETON, André: La Revolution Surrealiste, París, 15 de marzo de 1928, pp. 20-22.
} 
reconociendo que las cualidades de Picasso son propias, anteriores a ellos, y, siguiendo a Michel Leiris ${ }^{17}$, nunca surrealistas, pese a todo. Ello no fue tarea fácil. Vio como Picasso determinó una percepción renovada, basada en la independencia de la experiencia plástica, que, en su opinión, superó en mucho a los planteamientos de Matisse en sus cinco interpretaciones de Cabeza de Jannette, pues éste no relativizó la percepción del espectador; y la distribución plástica de sus obras más avanzadas, como Le Tiaré, ya se dio en obras de Picasso de 1909.

El planteamiento de Fernando Martín ${ }^{18}$ tiene un fundamento intelectual de largo alcance. En su opinión, Picasso suplió en estas obras lo psicológico precubista, los planteamientos cubistas y el problema de la corporeidad, con un interés desmedido por la expresividad y por los volúmenes superlativos conformados por las cualidades de un nuevo material plástico, el yeso. Werner Spies ya tuvo en cuenta el interés de Picasso por esta materia, confirmado en un comentario de éste a Brassai ${ }^{19}$; sin embargo, lo relegó a cuestiones superficiales y no lo vinculó, como Fernando Martín, a la propia configuración y a la expresividad del volumen. Su afirmación fue fundamental para comprender la naturaleza y la carga de sentido de las Cabezas de María Teresa Walter expuestas en el Pabellón de España de la Exposición Universal de 1937:

“...El tema va sufriendo una serie de transformaciones, cuya metamorfosis continua tiene su base en la insistente investigación expresiva, mediante el distinto potenciamiento de la forma. Así, de la helénica y pensativa Cabeza de mujer antes mencionada, se pasa a un segundo estado donde los rasgos faciales se acentúan en abultamientos que llegan a alcanzar autonomía propia" ${ }^{20}$.

A ello añadió que "El tema es lo de menos, lo importante es la manera de abordarlo, creando un lenguaje expresivo a partir de la deformación, cuya irregular asimetría le concede un particular poder expresivo" ${ }^{21}$.

Con distinto criterio que Werner Spies, Fernando Martín estableció la siguiente relación:

"La manera de concebir la figura, así como el tratamiento de las masas con su especial atención puesta en la textura, sigue muy de cerca a algunas de las esculturas de Matisse, cuyas analogías formales son bastantes parecidas, recuérdese por ejemplo su Janette $V$ de 1900-1931 o su Tairi con collar de 1930. Por otra parte resulta también paradójico comparar estas cabezas picassianas con las

\footnotetext{
${ }^{17}$ LEIRIS, Michel: “Toiles récentes de Picasso”; en Documents, París, 1930, pp. 57-70.

18 MARTÍN MARTÍN, Fernando: op. cit., p. 93.

${ }^{19}$ BRASSAÏ: Conversations avec Picasso, París, 1964. Méjico, Turner, Fondo de Cultura Económica, 1997, pp. 31 y ss.

${ }^{20}$ MARTÍN MARTÍN, Fernando: op. cit., p. 94.

${ }^{21}$ Ibidem, p. 95.
} 
realizadas años más tarde por Giacometti, donde un tallo grácil y filiforme a la manera de cuello, nos conduce a unas testas que sin abandonar sus formas anatómicas ofrecen una desgarradora expresividad" 22 .

De ese modo, insertó la serie en un proceso evolutivo concreto y la relacionó con otros grandes escultores del momento. La secuencia adquirió así un tinte estilístico determinado, ajeno a la naturaleza formal que pudiera corresponderle como categoría previa.

Juan Maldonado defendió otra posibilidad, vinculada a la capacidad lúdica de Picasso $^{23}$. Pensó que los cuellos alargados y las estructuras faciales con masas continuas en dos unidades superpuestas de las cabezas de María Teresa Walter y otras de la época, proceden de las llamadas sombras chinescas o juegos de luces y contraluces generados con sus manos sobre las paredes de los amplios espacios del Castillo de Boisgeloup. Las sombras proyectadas originarían los perfiles, según la equivalencia del brazo con el cuello y del juego de los dedos superpuestos con la estructura nariz frente.

La propuesta de Juan Maldonado, intuitiva, sorprendente, muy en consonancia con el carácter del artista, puede parecer aventurada, especulativa, aún así, el reconocimiento de la personalidad de Picasso, el predominio de la fantasía creadora en las esferas de las formas y las técnicas en la aplicación de su sistema sobre las más variadas referencias formales, y la similitud de las sombras con los perfiles de los grandes volúmenes que modeló en Boisgeloup, aconsejan, en principio, valorarla y tenerla en cuenta, sea como posible procedimiento o como medio auxiliar para cualquiera de las opciones propuestas. El método explicaría las relaciones entre las cabezas de las distintas series y los puntos en común con las obras aisladas.

\section{LA REFERENCIA NATURAL Y EL BELLO CLASICISMO DE CABEZA DE MARÍA TERESA WALTER I, EN 1931.}

Picasso reconoció que la referencia para la aplicación de su sistema, o, de haber varias, entre ellas, la primera, fue el natural. Werner Spies lo interpretó como la evolución de la individualización hasta la forma como signo de valor general. Esto implica, según él, un concepto de tiempo que oscila entre la destrucción y la persistencia.

\footnotetext{
22 Ibidem, p. 95.

${ }^{23}$ MALDONADO, Juan: Picasso único. Juicio a un genio en rebeldía, Málaga, Editorial Arguval, 2003, pp. 173-174.
} 
Tal principio determinó la objetivación de Cabeza de María Teresa Walter $I^{24}$, de la que Werner Spies dijo que su armoniosa belleza recuerda a la estatuaria clásica del Templo de Júpiter en Olimpia, de principios del siglo V a. C, y su rostro melancólico y abatido al Pensador de Rodín, en 1886. La belleza clásica es indiscutible.

Para Werner Spies, la nitidez de la forma, la pronunciada nariz, las mejillas redondeadas y, sobre ello, el pelo recogido en haces diferenciados y masivo y alisado en las superficies de los potentes volúmenes, propician la individualización y producen la simplificación y la concentración.

La cabeza apoya directamente en la base del cuello y experimenta una suave inclinación lateral y delantera. Así, presenta por delante la parte superior de la frente y obliga al espectador a corregir su posición hasta un punto de vista inferior que le permita establecer un nuevo rayo central de visión respecto del rostro. El tratamiento anatómico, preciso, veraz, y bien simplificado, remite al natural.

Las características del modelado, homogéneo, pulido, brillante, contribuyen a la depuración esencialista, vinculada a la proyección de conceptos intelectivos de raíces clásicas. En consecuencia, la simplificación y la monumentalidad de los volúmenes y de las superficies lisas y prietas son el toque de distinción que procede de los modelos de su pintura.

Esa configuración, y su testimonio, indican que Picasso utilizó dos referencias simultáneas, el natural, esto es, los rasgos físicos de su amante, y la estatuaria griega del estilo severo, que ya había sido una de sus fuentes en el Retrato de Fernande Olivier, en París, en 1906.

\section{EL SEGUNDO ESTADO, CABEZA EN RELIEVE DE MARÍA TERESA WALTER I, EN 1931}

La Cabeza en relieve de María Teresa W alter $I^{25}$ es el segundo estado de la aplicación iniciada con Retrato de María Teresa Walter I. La analogía formal con ésta es tan evidente que la identificación procede en el primer impacto visual. Picasso retrató a la joven de perfil y con los mismos criterios que en el primer retrato de bulto redondo. La adaptación al soporte y la disposición natural del sistema hacia las variaciones plásticas propiciaron novedades

\footnotetext{
${ }^{24}$ Colección Particular. Yeso, 53 x 49’5 x 37’5 cm; Bronce, 50 x 31 x 27 cm. Kahnweiler y Brassaï (KB) 46; Werner Spies (WS) 128.

${ }^{25}$ Colección Particular, original en yeso, 69 × 60 × $10 \mathrm{~cm}$, WS 130. Museo Picasso, París, ejemplar único en bronce, 68’5 x 59 x 8 cm. KB 62; WS 130; MPP 297.
} 
formales, en principio, casi imperceptibles; en definitiva, fundamentales para la progresiva transformación de los siguientes estados.

La posición lateral del rostro asume la condición del plano que condiciona la alteración proporcional de los volúmenes. Mejilla y pómulo adquieren cierta autonomía por la sobre dimensión, compensada por la masa del pelo, simplificada, orgánica y pesada, recogida, fijada detrás de las orejas y caída por la inercia. La posición frontal del ojo procede de la visión múltiple de época cubista. La irregularidad del tratamiento perimetral del soporte, sobre todo en el lado derecho del relieve, hacia el que mira el perfil izquierdo de María Teresa, aporta un aire desenfadado, idóneo para el origen difuso del cuello, abierto, ligero.

Picasso mantuvo los principios figurativos, la solidez técnica, el énfasis de bloque y la belleza formal del primer estado, basado en la referencia natural; mostró inquietudes en el desarrollo de los volúmenes en concordancia con las propuestas orgánicas de Cannes, Dinard y París; recurrió a planteamientos de estirpe cubista con la intervención de dos rayos centrales de visión; y, con todo ello, inició el camino en el que maduró las esculturas de María Teresa Walter para el Pabellón Español en la Exposición Internacional de París.

\section{EL BUSTO DE MARÍA TERESA WALTER, EN 1931, UNA DE LAS ESCULTURAS DEL PABELLÓN DE ESPAÑA EN LA EXPOSICIÓN INTERNACIONAL DE PARÍS, EN 1937}

El Busto de María Teresa Walter ${ }^{26}$ es el tercer estado de la aplicación y una de las tres esculturas vaciadas en cemento para el exterior del Pabellón de España en la Exposición Internacional de 1937. Perdida la réplica original en cemento, se conserva otra posterior en este material ${ }^{27}$.

Como en los anteriores, Picasso partió de soportes distintos, esta vez por la asunción del busto que amplía las bases del retrato de bulto redondo.

Las irregularidades en el encaje de los pechos respecto del eje y la anchura de la caja torácica, debidas a las dimensiones de ambos, semiesférico el derecho, en cuarto de esfera y corroído en la parte inferior y lateral izquierda éste, en consonancia con los huecos de los hombros, resueltos con criterios informalistas y texturas orgánicas, contrasta con la simplificación básica de aquélla y el cuello, atentos al natural, estilizados, ascensionales. La

\footnotetext{
${ }^{26}$ Colección Particular, original en yeso 78 x 46 x 48 cm; Museo Picasso, París, ejemplar único en bronce, 78 x 44'5 x 54 cm. KB 64; WS 131; MPP 298.

${ }^{27}$ Museo Picasso, París, cemento, 78 x 44’5 x 50 cm. MPP 299.
} 
rugosidad de las texturas de ese eje armoniza con la de los volúmenes inferiores y propone una alternativa eficaz al tremendo claroscuro de las superficies orgánicas laterales y de la parte inferior del pecho izquierdo.

La organización de los volúmenes del lado izquierdo del rostro coincide con la de Cabeza en relieve de María Teresa Walter I. La adecuación a la forma tridimensional determina la extensión circular de las superficies autónomas de éste, que, por ese motivo, pierden tal condición en beneficio del conjunto. Lo mismo sucede con el ojo izquierdo, que conserva la proyección oval y se extiende hacia delante perdiendo la frontalidad bifocal inicial.

El énfasis y la simplificación pareja de los volúmenes de la nariz y el pelo, resueltos en extensión, de modo unitario y con calidades compactas, apenas animadas en el caso de éste por unas incisiones superficiales en el sentido del giro que ajusta la masa al casco craneal, asumen la condición autónoma de la unidad nariz frente, ensayada con éxito en la talla en madera vista Busto de mujer, en Boisgeloup, en 1930.

El lado derecho de la escultura se presenta como un eficaz complemento en la conversión del busto redondo. Su aspecto inicial es más simple; sin embargo, los cálculos complementarios muestran una reflexión plástica compleja, tendente a la compensación de los volúmenes en zonas concretas y en el planteamiento conjunto. La contención del volumen de la mejilla, con superficies menos rugosas, aporta una insinuación de la referencia natural y el tratamiento clásico del primer estado; y la depresión sobre la mejilla y la reducción a la mínima expresión de la masa de pelo ajustada al casco craneal, lisa y velada, sitúan al ojo, la frente y la nuca, en un nivel inferior al de la unidad autónoma de la nariz frente y el lado resultante.

Los dos niveles disocian en unidades compactas los elementos físicos propios de María Teresa Walter, y de ese modo, el organicismo propio de la mujer es sustituido por la presencia plástica de dos bloques bien definidos y autónomos; aunque relacionados por la masa básica de la parte trasera y la dirección de los movimientos de cada uno.

\section{DESARROLLO DESPROPORCIONADO DE LOS VOLÚMENES Y NUEVO ORDEN, CABEZA EN RELIEVE DE MARÍA TERESA WALTER II, EN 1931}

La cronología propuesta por Christine Piot para Cabeza en relieve de María Teresa Walter $I^{28}$, en 1931-1932, es consecuente con la relación establecida por Werner Spies entre Cabeza de

${ }^{28}$ Colección Particular, original en yeso, $20 \times 16$ x 9 cm, WS 129. Ejemplar único en bronce, $20 \times 16 \times 9 \mathrm{~cm}$, fundido a la cera perdida por C. Valsuani, KB 61; WS 129. 
María Teresa Walter II y dos dibujos escultóricos, titulados, Estudios para una escultura ${ }^{29}$, firmados el día cinco de diciembre de 1931.

El problema pendiente de resolución es el de la cronología de la escultura, pues la secuencia exacta de la aplicación en función de la evolución formal apreciable en las obras citadas, incluye al relieve entre los dibujos y ésta. Es más, la analogía entre el relieve y los dibujos es tal que no puede descartarse su origen como materialización en tres dimensiones de unos de ellos o, incluso, de ambos a la vez.

Si se admite para la escultura la fecha de 1931, mantenida por Piot y Spies, y se considera derivada de los dibujos, el margen de ejecución es escaso, entre los días seis y treinta y uno de diciembre de ese año. Ajustado esto, el relieve, análogo a los dibujos escultóricos, por lo tanto, anterior a la escultura, tendría un margen aún más reducido, en los días inmediatos al cinco de diciembre.

La composición está supeditada al plano, con tendencia rectangular mas irregular en la proyección de los contornos. La cabeza, dispuesta sobre el perfil derecho, está formada por tres volúmenes emergentes y dos esferas intermedias que completan el sentido. La relación, a medio camino entre la articulación volumétrica y la estructuración constructiva, es muy particular.

Aquéllos forman la barbilla, en perspectiva angular, y la mejilla derecha; la nariz; y el pelo y la frente, y funcionan como unidades autónomas equiparables a las de los dibujos escultóricos Figura I a XIII, en Dinard, en 1928. La única diferencia está en el punto de unión común al soporte; no obstante, la condición de altorrelieve minimiza esta circunstancia y posibilita la autonomía volumétrica y visual de cada unidad. Las pequeñas esferas también son autónomas. Su intervención en las depresiones intermedias es clave en la configuración. Una sobre la mueca con la que representó la boca, como complemento visual de ésta, desplazada hacia la mejilla, y en la base interior de la nariz; otra en el espacio resultante entre las tres unidades, sobre el pómulo, en el lado derecho de la parte superior interna de la nariz, y bajo el bloque del pelo y la frente, éste concebido con la forma de boomerang procedente de la referencia propuesta.

Cada unidad autónoma es en sí una abstracción orgánica sin más significado propio que el de la realidad informe. La configuración final depende de la relación de las formas, en un conjunto en el que las cualidades plásticas, basadas en el equilibrio de los elementos, masivos, contundentes, pesados, propiciado por la intervención de las esferas en

\footnotetext{
${ }^{29}$ Museo Picasso, París. 32’7 x 25’4 cm cada uno. MPP 1065 y 1067.
} 
puntos claves para la distribución de los empujes virtuales, destaca sobre la propia figuración.

\section{LA SEGUNDA ESCULTURA DEL PABELLÓN DE ESPAÑA EN LA EXPOSICIÓN INTERNACIONAL DE PARÍS: CABEZA DE MARÍA TERESA WALTER II, EN 1931}

Werner Spies destacó en Cabeza de María Teresa Walter $I I^{30}$ el carácter derivado de las simplificaciones del modelo original y el sentido compacto de la distribución de los volúmenes, que aseguran la particularidad de los espacios de cada forma y ello la nitidez del conjunto $^{31}$.

La composición parte de una base tronco piramidal, irregular, informe, en la que los pegotes de barro, visibles, crean un efecto rudo, descuidado, que establece dos diálogos, uno en lo relativo al claroscuro de las superficies; otro, en el contraste con la forma del cuello, emergente y sobre puesto, como si tal base fuese parte de un alma interior, descarnada, descubierta. El aspecto es el de dos tramos de un mismo soporte, el de un amplio cuello dividido en dos tramos por el distinto tratamiento de las texturas en el tercio superior. En conjunto, la composición queda así sobre elevada y, en cierto modo, estilizada.

Los perfiles, análogos, pese a ello, asimétricos, concuerdan con la estructura de Cabeza en relieve de María Teresa Walter II. La esfera superior cumple la misma función; la inferior no, en la escultura ocupa una posición similar a la primera esfera, como ojo izquierdo de la figura. La distinta naturaleza del soporte, bulto redondo en vez de relieve, explica la variante en la relación de los volúmenes, pues la autonomía inicial de las unidades de éste, sólo supeditada a la condición del plano, imposible en el nuevo medio, depende aquí de la prolongación de la masa del eje, visible por el lado izquierdo de la escultura, en el que actúa, a la vez, como soporte, y como núcleo originario de los volúmenes expansivos que sustituyen a las unidades autónomas, cuyas relaciones visuales mantienen respecto de aquél.

La continuidad de la extensión que forma la mejilla izquierda sobre el núcleo básico de la prolongación del cuello, voltea en la barbilla hacia el lado contrario, en el que la

\footnotetext{
${ }^{30}$ Colección Particular, París, original en yeso y madera, 87 x 30 x 45 cm; Colección Particular, París, modelo intermedio en yeso; Museo Picasso, París, ejemplar único en bronce, 86 × 32 x 48`5 cm; Edición de dos ejemplares en bronce, Valsuani, 1973, 86 × 32 × 48’5 cm; Museo de Antibes, escultura expuesta en el Pabellón de España en la Exposición Internacional de París, en 1937, 86 x 32 x 48`5 cm. KB 59 y 60 (edición de dos ejemplares en bronce); WS 132 (todas); MPP 300 (edición de un ejemplar en bronce).

31 Spies, Werner: La escultura de Picasso; Op. Cit. p. 157.
} 
forma orgánica asciende sobre la depresión que la separa de la nariz y la esfera del ojo. El modelado, masivo, en forma de ese con amplia base, oculta el núcleo cilíndrico, perceptible, con un considerable esfuerzo visual, en la zona de la depresión intermedia con la nariz y el ojo, en la que el claroscuro, dispuesto con habilidad, asume la fuerza expresiva que distrae la apreciación del carácter constructivo de la configuración.

La nariz es el segundo volumen en extensión y, como el anterior, tiene dos ángulos visuales distintos, uno desde cada perfil. En el izquierdo, la extensión orgánica parece un apéndice simple, que no es, ni por apéndice, ni por simple; tal se comprueba en el lado opuesto, en el que la rectitud torna en movilidad con el modelado contrapuesto de la base, cóncava, en relación con la flexión convexa de la extensión anterior. Su aspecto, desarrollado, masivo, es proporcional al desarrollo de ésta.

El pelo, ajustado al casco craneal, simplificado, reducido al resalte de la tercera extensión, como en Cabeza en relieve de María Teresa Walter II, forma un bloque compacto en función de los imperativos del bulto redondo. La simplificación, extrema, y la unificación de las superficies, con la salvedad del intenso contraste de las texturas de la base y el cuello, aumenta la sensación de bloque que simula la concepción modular fundamentada en la correspondencia de las unidades, sustituidas por sus equivalentes en extensión.

Puede concluirse que la simplificación, según las zonas muy intensa, esquemática, casi mecanizada, aportó un nivel intermedio en el proceso plástico de deformación formal que caracterizó a los siguientes estados; aunque éstos no estén aún precisados y ni siquiera intuidos en su verdadero alcance, al menos en lo que refiere a la realidad material de la escultura. Otra cosa es el alcance intelectivo de ésta, que permitió a Picasso afrontar el paso decisivo en la evolución de la serie.

\section{LA ESCALA MONUMENTAL DE LA TERCERA ESCULTURA EN EL PABELLÓN DE ESPAÑA DE LA EXPOSICIÓN INTERNACIONAL DE PARÍS: CABEZA DE MARÍA TERESA WALTER III, EN 1931}

Picasso dio el paso definitivo en la tercera escultura que mostró en el Pabellón de España de la Exposición Internacional de París, Cabeza de María Teresa Walter III ${ }^{32}$. Werner Spies

\footnotetext{
32 Museo Picasso, París, original en yeso y madera, $128^{\prime} 5$ x 54’5 x 62’5 cm; Museo de Arte Moderno, Nueva York, modelo intermedio en yeso; Museo Picasso, París, ejemplar único en bronce, fundido antes de 1934, $128^{\prime} 5$ x 54'5 x 62’5; Museo de Antibes, escultura expuesta en el Pabellón de España en la Exposición Internacional de París, en 1937, 128’5 x 54’5 x 62’5 cm. KB 63 (ejemplar único en bronce); WS 133 (todas); MPP 301 (original) y 302 (ejemplar único en bronce).
} 
expuso $^{33}$ que Picasso trató los elementos, abultados, redondeados, un tanto autónomos, como piezas desmontables que se agrupan según las proporciones naturales. En esa agrupación, percibió la ascendencia de obras cubistas de 1906; la posible influencia de una Máscara japonesa publicada en 1928; y una interpretación relativa del punto de vista del espectador, que comparó con las esculturas de Matisse, Jeannette y Le Tiaré, en las que no encontró la misma intensidad por la superior independencia de la experiencia plástica en el modelado de Picasso. Planteada ésta, estableció el origen del procedimiento en 1926, identificó la estructura de la nariz frente en obras de ese año ${ }^{34}$, y propuso como antecedentes inmediatos de las esculturas una serie de dibujos y pinturas ${ }^{35}$ propias de 1932.

En esta escultura, de carácter monumental, el tratamiento del cuello, cilíndrico, alargado, estilizado, realza los volúmenes de la cabeza, configurada a partir de las extensiones informes que, agrupadas, originan la cara. La homogeneidad de la masa de yeso (después bronce o cemento) asume la distribución de los volúmenes autónomos mediante las inflexiones de la materia en extensión, modulada por las depresiones que determinan tres zonas, todas orgánicas e informes, que aluden a la barbilla con las mejillas, la nariz frente y la cabeza con el pelo, éstos últimos ligeramente desplazados hacia detrás y abajo.

Las dos inflexiones que posibilitan la división en tres grandes bloques de una misma extensión, que prevalece desde cualquier perspectiva que sea contemplada o pensada, tienen distinta concepción e intenciones. La que modula los volúmenes informes de la cara y la nariz frente, frontal y con sentido cóncavo. El modelado ascendente está determinado por la diagonal superior y la proyección abocinada en ángulo, que posibilitan la referencia carnal y la prominencia adecuada para la alusión de la nariz.

En esa inflexión aparece un elemento figurativo clave para la interpretación del conjunto, la boca, modelada con criterio naturalista sometido a la simplicidad esencial acorde con las simplificaciones de los volúmenes orgánicos. Una incisión oval invertida, que representa el ojo, ocupa la zona en la que la extensión deriva en ambos sentidos, es decir, en el ángulo superior e interior de la inflexión abocinada.

La segunda inflexión separa la cabeza y el pelo de la cara y la nariz frente. Es una depresión vertical y ondulada, marcada por una incisión que potencia el efecto. La continuidad de la res extensa queda así atenuada, y el bloque, en forma de boomerang masivo, pesado, alterado en la parte superior por una incisión que corrige el perfil, se distancia tanto por la abstracción radical de los elementos a los que alude, como por la

\footnotetext{
33 SPIES, Werner: op. cit., pp. 156 y 157.

${ }^{34}$ Z VII, 5; Z VII, 22.

35 Z VII, 346; Z VII, 378.
} 
autonomía que le proporciona tal recurso según las características de las unidades autónomas, en realidad, inexistentes en la figura.

La constitución de la elevada base cilíndrica y la continuidad de ésta con la prolongación del cuello, apenas diferenciados por una matización casi imperceptible de las texturas, rugosas, espontáneas, directas, deriva de Cabeza de María Teresa Walter II. Las extensiones están dispuestas en consonancia con las unidades autónomas de Cabeza en relieve de María Teresa Walter II, y está clara la experiencia en la proyección en extensión derivada y la consecuente unidad material de la variante inmediata, la citada Cabeza de María Teresa Walter II.

La excelencia compositiva, fundamentada en la eficaz simplificación de los volúmenes informes de naturaleza orgánica y, sobre todo, en la espontánea monumentalidad de tal proceso de síntesis, propios del último estado de la serie, la convierten en una de las esculturas más importantes del catálogo de Picasso, y, sin lugar a dudas, en uno de los prototipos fundamentales en la evolución de la escultura contemporánea.

\section{EL VOLUMEN EN EXTENSIÓN, CONCLUSIONES SOBRE LAS CABEZAS DE MARÍA TERESA WALTER}

La secuencia propuesta por Werner Spies sobre la posible ascendencia de la estructura de las cabezas de María Teresa Walter, es un tanto confusa. En cierto modo, se contradijo, pues propuso el origen en los proyectos cubistas, después afirmó que el modelo fue la Máscara japonesa, publicada en 1928, y finalmente que esa continuidad o integración de la nariz y de la frente reapareció en la obra de Picasso en 1926. Es elemental, si es una formación propia de 1907 que retomó en 1926, jamás puede proceder de una referencia ajena en 1928.

Sí fue un acierto estimable de Spies la secuencia de la serie desde Cabeza de María Teresa Walter I hasta Cabeza de María Teresa Walter III, incluidos, entre ambas, los estados con otros títulos y los relieves. Lo que Spies no explicó fue la naturaleza del sistema creativo de Picasso, y, por ello, se le escapó la posible participación de dos o más referencias de modo simultáneo, como objetivo que las canaliza; y la doble posibilidad de las definiciones formales y de la creatividad técnica, lo cual eleva a niveles intelectivos la comprensión de su obra. Esto lo limitó a destacar las cualidades formales y a relacionarlas 
con aquéllas que las comparten en un elevado número de dibujos y pinturas, que representó con dos ejemplos concretos.

La primera Cabeza de mujer de Fernando Martín es Cabeza de María Teresa Walter I. Él planteó la evolución de la iconografía y se dio cuenta de una característica común para los estados escultóricos y las pinturas en las que siguió el desarrollo de la serie, la deformación en la que los rasgos faciales se simplifican en grado extremo al servicio de la expresión, determinada por la nariz prominente que determina la mayor parte del rostro. Ello las aparta de la belleza ortodoxa, clásica, del primero de los estados. Esa expresión prioritaria procede, según Martín, tanto de la distorsión de los volúmenes como de la exaltación de la materia, de manera que el tema no es más que un pretexto para establecer nuevas posibilidades visuales en campos de vanguardia. Excelentes observaciones.

Una de las aportaciones más importantes de Fernando Martín sobre estas esculturas fue su insistencia en la factura anterior a los vaciados en cemento para la Exposición Universal de 1937, y después, en la situación jerarquizada en el exterior del Pabellón de España. Esto permite equipararlas a los proyectos escultóricos Bañista I a XX, en Cannes, en julio de 1927, concebidos como grandes realizaciones plásticas que le hubiera gustado disponer, alineadas, en un paseo marítimo. La intención monumental de Picasso fue la misma en ambos casos.

Otra, fundamental, fue la consideración de un tratamiento de las masas y las texturas afines a las practicadas por Matisse desde la segunda década del siglo XX. Fernando Martín le adjudicó a ello ciertos parecidos formales, que se mantienen a simple vista y se diluyen cuando se profundiza en la concepción plástica.

Los valiosos juicios de Werner Spies y Fernando Martín ayudan a dilucidar la aplicación del sistema de Picasso propuesto. Spies dijo que la configuración de estas cabezas procede del arte cubista; pero, por más que se intente, no se consigue establecer ningún punto de inflexión entre la técnica aditiva constructivista, propia del cubismo, en la que los elementos se superponen con sentido tectónico, y la res extensa del modelado continuo de estas obras.

La primera referencia de algo que permita la indagación formal en este sentido, está, como en la escultura en hierro y los proyectos escultóricos de obras modeladas de 1929 a 1931, en las aplicaciones del sistema sobre la referencia que constituyen las leyes de la composición fotográfica, ello en función de modelos clásicos o del natural, indistintamente, en 1917 a 1927. La cronología planteada y el origen de la estructura nariz 
frente antes de la actividad en Boisgeloup, relegan a un papel secundario la posible aportación de las proyecciones de sombras propuestas por Juan Maldonado.

En pinturas como Madre e bijo ${ }^{36}$, en Dinard, en 1922; Retrato de Olga (Olga pensativa $)^{37}$; y Cabeza de mujer ${ }^{38}$, las dos en París, en 1923, un fino trazo representa de una vez una ceja y la nariz. Son figuraciones conformes con el natural y cada elemento guarda la proporción que le corresponde respecto del otro y de los demás de la cara; sin embargo, sus cualidades formales, una vez propuestas como referencias de una nueva aplicación del sistema, basada, como aquí se defiende, en las propiedades intrínsecas de la forma previa, sí permiten la evolución de las prominencias expresivas en el sentido que las propuso Fernando Martín.

De ellas se pasaría a otros estados, como tales o asociados a otros en composiciones mayores, tal fue habitual en Picasso desde las épocas azul y rosa y en pinturas trascendentales en la orientación de las vanguardias, por ejemplo, Las Señoritas de Aviñón y Guernica ${ }^{39}$, es el caso de Mujer sentada, en París, en 1927; Cabeza, en 1929; las citadas La lectura, El salvamento y El sueño, en 1932; y, entre ellas, en el lugar que cronológicamente le corresponde, la talla en madera vista Busto de mujer, en 1930. Ésta, como Pareja, conserva el sentido cerrado del bloque de madera.

De este desarrollo se pueden sacar varias conclusiones. La más importante es que las cabezas del Pabellón Español de la Exposición Universal de París, en 1937, son estados maduros y finales de la aplicación del sistema de Picasso sobre dos referencias simultáneas, las que suponen la interpretación prohelénica, admitida por Spies y Martín, de Cabeza de María Teresa Walter I, y de la anterior indagación formal, representada por el Busto de mujer que talló en 1930, cuyos resultados persiguió con el nuevo modelo, circunstancia que éstos no citaron.

Esta nueva y más compleja aplicación del sistema es la que produjo las relaciones propuestas por Spies, a las que se pueden añadir otros muchos estados en dibujos y pinturas, los más significativos los que aparecen en El escultor, pintura citada, en 1931, que es correlativo a Busto de mujer, y en un documento del Museo Picasso de París ${ }^{40}$, éste nexo de unión con una variante que ya vimos en los proyectos escultóricos de años anteriores, en la que los principales volúmenes están concebidos con autonomía y después montados según los principios constructivos.

\footnotetext{
${ }^{36}$ Museo de Bellas Artes, Baltimore. Óleo sobre lienzo, 100 x 87 cm. Z IV, 371.

${ }^{37}$ Museo Picasso, París. Papel y lápiz sobre papel, 104 x 71 cm. Z V, 38; MPP 993.

38 Museo de Bellas Artes de Bridgestone, Tokyo. Óleo sobre lienzo, 46 x 38 cm. Z V, 45.

${ }^{39}$ Centro de Arte Reina Sofía, Madrid.

40 MPP 1065.
} 
En lo referente a la secuencia concreta de la serie de esculturas sobre María Teresa Walter habría que señalar la posibilidad de un primer estado, lo más probable es un dibujo, tomado del natural, o, como posibilidad alternativa afín, en éste como referencia directa para la primera aplicación, simultánea a la griega clásica, cuyo resultado fue Cabeza de María Teresa Walter I. A partir de ahí, el proceso tuvo una intención plástica basada en dos principios, la continuidad del modelado en extensión, fundamentada en la organización de bloques complementarios, y la adición de unidades independientes, en los dos casos, en función del doble juego establecido entre la fuerza de las abstracciones orgánicas y la condición figurativa que prevalece.

El segundo paso fue Cabeza en relieve de María Teresa Walter II, próxima al primer estado escultórico; mas tendente a la adaptación de los volúmenes al plano del soporte, circunstancia que motivó el protagonismo de los elementos físicos correspondientes. El aumento proporcional de éstos fundamentó la variante Busto de María Teresa Walter. Picasso llevó el nuevo orden al plano en Cabeza en relieve de María Teresa Walter II, y las condiciones del soporte fueron de nuevo fundamentales, pues la fuerza de las extensiones resultó equivalente a la de las unidades autónomas de los dibujos escultóricos de Dinard, en 1928. Esa configuración mediante unidades fue aparente no real. Sirvió de referencia para los volúmenes extensivos de Cabeza de María Teresa Walter II, y éstos, a su vez para los de la brillante abstracción orgánica que culminó el proceso con Cabeza de María Teresa Walter III.

\section{OTRAS OPCIONES, POSIBLE CABEZA EN RELIEVE DE MARÍA TERESA WALTER I A IX, EN 1933 Y 1934}

Los nueve relieves en miniatura titulados Cabeza de mujer, en 1933 a 1934, aparecen identificados en el catálogo de Christine Piot y Werner Spies por aspectos concretos de la composición, como los perfiles, o el busto.

Las características de modelado son muy distintas entre sí; sin embargo, la distribución de volúmenes, resaltes lineales o huecos indicativos de volúmenes virtuales, coinciden con la estructura básica de la cabeza de María Teresa Walter. Por ese motivo, la identificación no es aventurada.

La Posible cabeza en relieve de María Teresa Walter $I^{41}$, sería la tercera si se confirmase la identidad, y así sucesivamente con las otras ocho, hasta la número once. El perfil izquierdo está modelado mediante volúmenes lineales que resaltan sobre las depresiones del

\footnotetext{
${ }^{41}$ Museo Picasso, París. Yeso, 32’5 x 23 x 7’5 cm. KB 87; WS 138; MPP 306.
} 
soporte. Tal contraste crea un potente claroscuro, que produce una sensación cercana a la de las tallas en huecorrelieve. Las unidades positivas, barbilla mandíbula, nariz frente y cabeza pelo, funcionan como unidades autónomas completadas por los volúmenes virtuales propuestos por las zonas intermedias en hueco. En el caso de la primera, la diagonal interior se prolonga hasta la segunda unidad, circunstancia que propicia la continuidad. La boca, la extensión esférica que alude al pómulo y el ojo, oval e invertido, con rabillo, y frontal, actúan como elementos intermedios fundamentales en la configuración.

El sentido lineal y la fuerza de los espacios vacíos es mucho mayor en Posible cabeza en relieve de María Teresa Walter $I I^{42}$. Tanto que el perfil izquierdo es un auténtico huecorrelieve, que sólo presenta un volumen cercano al positivo en el perfil exterior de la nariz. La definición de la silueta contrasta con la abstracción de las proyecciones lineales, abundantes, laberínticas, en el interior.

El cambio de perfil, ahora el derecho, diferencia a la Posible cabeza en relieve de María Teresa Walter $I I I^{43}$. Es posible que se trate del tercer estado de una misma aplicación que los anteriores. La relación de volúmenes lineales sobre el plano produce la relación de espacios vacíos que propone volúmenes virtuales. En conjunto, la composición presenta una forma más redondeada y con menor desarrollo de la nariz frente, que mantiene las directrices, la mueca de la boca y el sentido curvilíneo de la caída del pelo, ajustado al casco craneal. El fondo rugoso del soporte, abultado por la adición de esferas de distintos tamaños, aporta un sentido decorativo peculiar.

La cuarta variante, Posible cabeza en relieve de María Teresa Walter $I V^{44}$ mantiene las características de la anterior, reducidas por el menor tamaño y modificadas por la supresión del cuello, que aumenta el sentido envolvente de la silueta y el claroscuro del espacio intermedio del pelo, y la imprecisión del modelado positivo de la zona de la boca y el negativo de la mejilla. La inscripción en un tondo, trabajado en negativo, y doblado por una secuencia circular de medias esferas anexas, remite a la estética de la orfebrería de los pueblos hispanos del sur en segundo milenio antes de Cristo, incluido el horizonte orientalizante de época tartésica.

El siguiente estado de la misma aplicación, Posible cabeza en relieve de María Teresa $W$ alter $V^{45}$, mantiene el perfil derecho. La configuración recuerda la estructura del segundo estado, invertido y modificado por la mayor presencia de los volúmenes positivos, que

\footnotetext{
42 Colección Particular. Yeso, 59’5 x 57 x 6’8 cm. KB 86; WS 139.

${ }^{43}$ Colección Particular. Yeso, 22 x 16’5 x 5 cm. KB 93; WS 140.

${ }^{44}$ Colección Particular. Original en yeso, diámetro del tondo 11'5 cm. KB 88; WS 146.

45 Museo Picasso, París. Yeso, 19 x 16 x 4’5 cm. KB 92; WS 141; MPP 307.
} 
mantienen el carácter lineal, y no el predominio de las zonas vacías y los volúmenes virtuales, reducidos a complementos, reforzados con el huecorrelieve exterior y perimetral que realza el modelado de la figura.

El último estado de la aplicación es Posible cabeza en relieve de María Teresa Walter $V T^{46}$, como todos los anteriores en 1933. La simplificación lineal la reduce a la presencia de una silueta, incompleta en dos puntos, los que separan el inicio y la caída de la línea ondulada del pelo, cuyo interior manifiesta el plano del soporte, circunstancia que convierte el interior en un huecorrelieve virtual, completado por la silueta análoga y semiesférica del ojo, completada por una pequeña esfera interior, alusiva al globo ocular.

La obra titulada Posible cabeza en relieve de María Teresa Walter VII ${ }^{47}$, en 1933, procede de una aplicación distinta, la segunda identificada hasta este momento. La hipotética referencia pudo ser el cuarto estado, del que conserva el sentido circular, aplicado al volumen y no al tratamiento del formato, éste rectangular y simple, análogo a las variantes sexta, octava y novena. Presenta dos novedades formales, el giro semifrontal de la composición, en el que aún impera el lado derecho de la figura; y el modelado real, positivo, de casi toda ella, con la salvedad de un entrante negativo en forma de busto en diagonal interior, que ocupa la parte superior del lado predominante, en el que se inserta una potente esfera, con la que alude al ojo.

Más difícil es asegurar si la Posible cabeza en relieve de María Teresa Walter $V I I I^{48}$, en 1933-34, es la segunda variante de la segunda aplicación, o forma parte de una tercera. La disposición frontal dificulta la identificación. La composición oval y envolvente, el fuerte claroscuro de los resaltes lineales que forman el pelo, el equilibrio establecido entre las partes modeladas y las trabajadas en hueco, la continuidad de la zona de la frente y las esferas de los ojos, remiten, como en el caso anterior, al cuarto estado, motivos suficientes para la consideración en ese sentido. El soporte, rectangular y con superficies lisas, pulidas y brillantes, concuerda con la séptima variante. La continuidad del resalte lineal y frontal de los pómulos, casi en régimen de simetría, actúa sobre la boca como una corrección óptica que aporta una falsa mueca, cuya sonrisa, irreal y un tanto forzada, modifica la expresión estable de todos los estados anteriores.

\footnotetext{
${ }^{46}$ Colección Particular. Ejemplar único en bronce, 16’5 x 14 cm. KB 85; WS 145.

${ }^{47}$ Museo Picasso, París. Original en yeso, 15 x 20’5 x 7 cm. KB 90; WS 144.

48 Colección Particular, original en yeso, $22 \times 14^{\prime} 5 \times 9 \mathrm{~cm}$, WS 142. Colección Particular, ejemplar único en bronce, $21 \times 14^{\prime} 5 \mathrm{~cm}$, fundido a la cera perdida por E. Robecchi, KB 91; WS 142.
} 
La mayor dificultad radica en la Posible cabeza en relieve de María Teresa Walter IX $^{49}$, en 1933-34. La posición frontal concuerda con el octavo estado; sin embargo, la configuración del busto, la relación de la figura con el soporte, la renuncia al esquema oval y los distintos principios armónicos, difieren en demasía. El trabajo lineal mediante finos resaltes verticales, envolventes y paralelos, que forman el pelo, asimétrico en la doble caída frontal; y la relación del resalte interior con la franja afín en hueco que lo separa de los volúmenes del rostro, recuerdan la brillante simplificación del sexto estado. El equilibrio entre los volúmenes reales y los vacíos virtuales en la configuración facial, no tiene nada que ver con la simplificación radical de aquél. El potente claroscuro originado por la cercanía de los resaltes de los pómulos, los ojos y la estructura de la nariz frente, proporcionada y respetuosa con los modelos naturales, no tienen equivalencia en ninguno de los ocho estados anteriores.

\section{Conclusiones}

De todo ello puede concluirse que las cuatro cabezas de María Teresa Walter fueron modeladas por Picasso antes del encargo de las autoridades de la Segunda República para el Pabellón de España de la Exposición Internacional de París del año 1937. Forman una serie con unidad plástica reconocible a partir de una referencia natural; y fueron vaciadas después en cemento bajo la dirección del propio Picasso, otorgándole éste el carácter monumental por el que fueron conocidas mundialmente.

El objetivo del vaciado en cemento y la inclusión de las cuatro cabezas en el perímetro del pabellón fue el apoyo expreso de Picasso a la causa del legítimo gobierno de España, constituido por la Segunda Republicana, decisión comprometida que no puede interpretarse como un hecho partidista, sino como la defensa de un marco de libertades que, según pensaba, sólo correspondía al pueblo español.

Las cuatro forman parte de una serie en la que significan y no tienen ninguna carga de sentido simbólico relacionada con la temática del pabellón ni con la guerra civil española ni la política. Son obras independientes en las que, como fue usual en Picasso, trabajó con fundamentos plásticos, estableciendo una serie de variantes a partir de las cualidades formales propuestas en la anterior, con lo que el artista recorrió una camino progresivo hacia la simplificación extrema y el fundamento de un nuevo orden estructural que la explica en sí misma.

49 Colección Particular, original en yeso, 29 x 25’5 x 7’5 cm, WS 143; Colección Particular, ejemplar único en bronce, $28^{\prime} 5$ x 25’5 x 8 cm, fundido a la cera perdida por E. Robecchi, KB 94; WS 143. 
Picasso trabajó en dos series distintas a partir de un retrato y apuntes del natural, una en relieve y la otra de bulto redondo. En los dos casos optó por el reconocimiento de una referencia natural, modelada con atención a principios clásicos descontextualizados y simplificados con extraordinaria eficacia y un sentido de la monumentalidad muy moderno, a partir de la cual optó por las deformaciones de las características originales en sendos procesos de codificación que las aproximaron a las abstracciones orgánicas y las bioformas, anticipando, por una parte, el sentido que seguirían las estéticas asociadas en la siguiente década; y, por otra, un orden estructural muy personal y característico, que fundamenta a la obra en sí.

Tanto las esculturas como los relieves presentan una secuencia ordenada y lógica en el sentido de la deformación o abstracción orgánica. Por ello, aunque cada uno de los estados tiene una identidad plástica definida y concreta y, por lo tanto, es plenamente autónomo, el modo idóneo de verlas es de forma conjunta, como serie, según la secuencia original completa.

La libertad creativa de Picasso se muestra en plenitud y sin límite alguno, tanto para el aprovechamiento de recursos plásticos con fundamentos clásicos en el primer estado, como para la utilización de recursos derivados del lenguaje cubista ya superado; de la mentalidad expresionista; del sentido del orden estructural constructivista; y de la capacidad de transformación y la ambigüedad surrealista. Picasso participó de todo ello y se anticipó a los movimientos artísticos indicados, conectando con todos de un modo muy personal y sin participar en ninguno. Su creatividad no tuvo límites, y además con ello se presentó como un antecedente directo para la mentalidad postmoderna propia de la década de los setenta del siglo XX.

La definición estructural nariz-frente es buena prueba de esa posición formal y plástica superior a los estilos, característica de la mayor parte de la producción artística de Picasso. Se trata de una codificación al límite de la abstracción, que adquiere sentido en la relación de los dos grandes bloques y los distintos elementos de cada una de las artes, y está dotada de una fuerte carga expresiva.

Picasso utilizó estructura nariz-frente en varias pinturas del año 1932, unas veces pronunciando los volúmenes con el sentido escultórico original; otras concibiéndolo de modo virtual, en hueco o mediante siluetas y contrasiluetas de estirpe cubista, utilizadas con distinto criterio y aprovechamiento. La actualización de los recursos y el dominio en la utilización de colores graduados tonalmente o planos, según los casos, aumentaron las posibilidades creativas desde la esfera de la producción técnica. 
Uno de los estados más avanzados de la serie en relieve le sirvió a su vez como nueva referencia para el desarrollo de otra serie, Muchacha delante de una caseta de playa, en la que experimentó junto a Dora Maar con técnicas mixtas en las que pintó sobre negativos fotográficos. Es claro ejemplo de cómo un estado puede ser una variante de una serie y a la vez la referencia o punto de partida para el desarrollo de otras, procedimiento que le permitió a Picasso desarrollar de modo simultáneo una gran variedad de posibilidades formales, con resultados unas veces consecuentes; otras en apariencia muy divergentes y sin aparente relación. En este ejemplo se ve muy bien que el punto de partida conceptual, como tal inmutable, asume la posibilidad de intervención de un nuevo factor experimental, que, una vez objetivado, puede convertirse en la siguiente referencia para una nueva aplicación del sistema creativo de Picasso, único en su planteamiento y siempre genial en sus soluciones. 


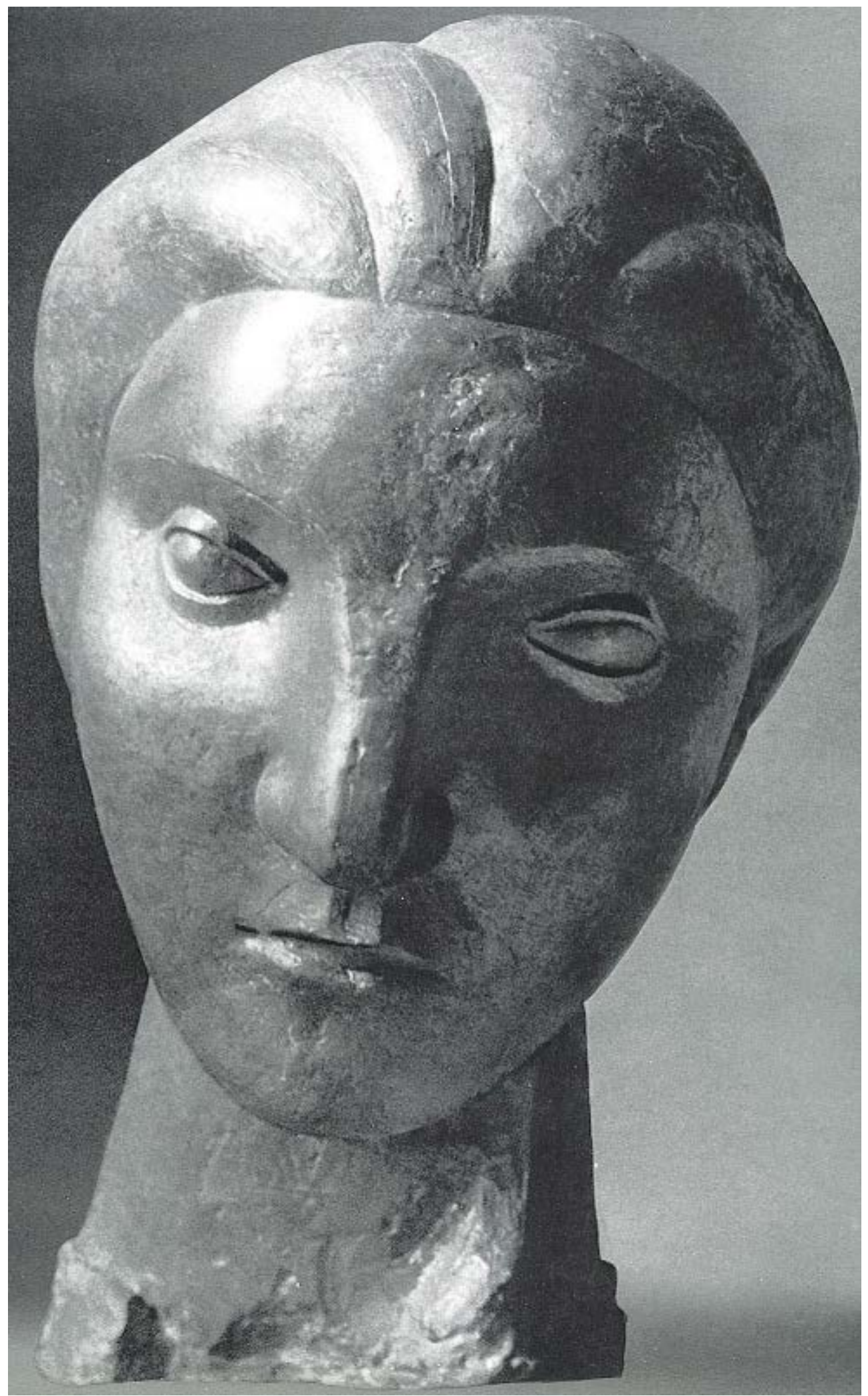

Lám. 1- Picasso, Cabeza de María Teresa Walter I, Boisgeloup, 1931.

(Brassï (Introduction Daniel-Henry Kahnweiler), Les Sculptures de Picasso. París, 1947)

La doble referencia, naturalista y de la escultura griega clásica, fueron los fundamentos básicos de la contundente simplificación formal. 


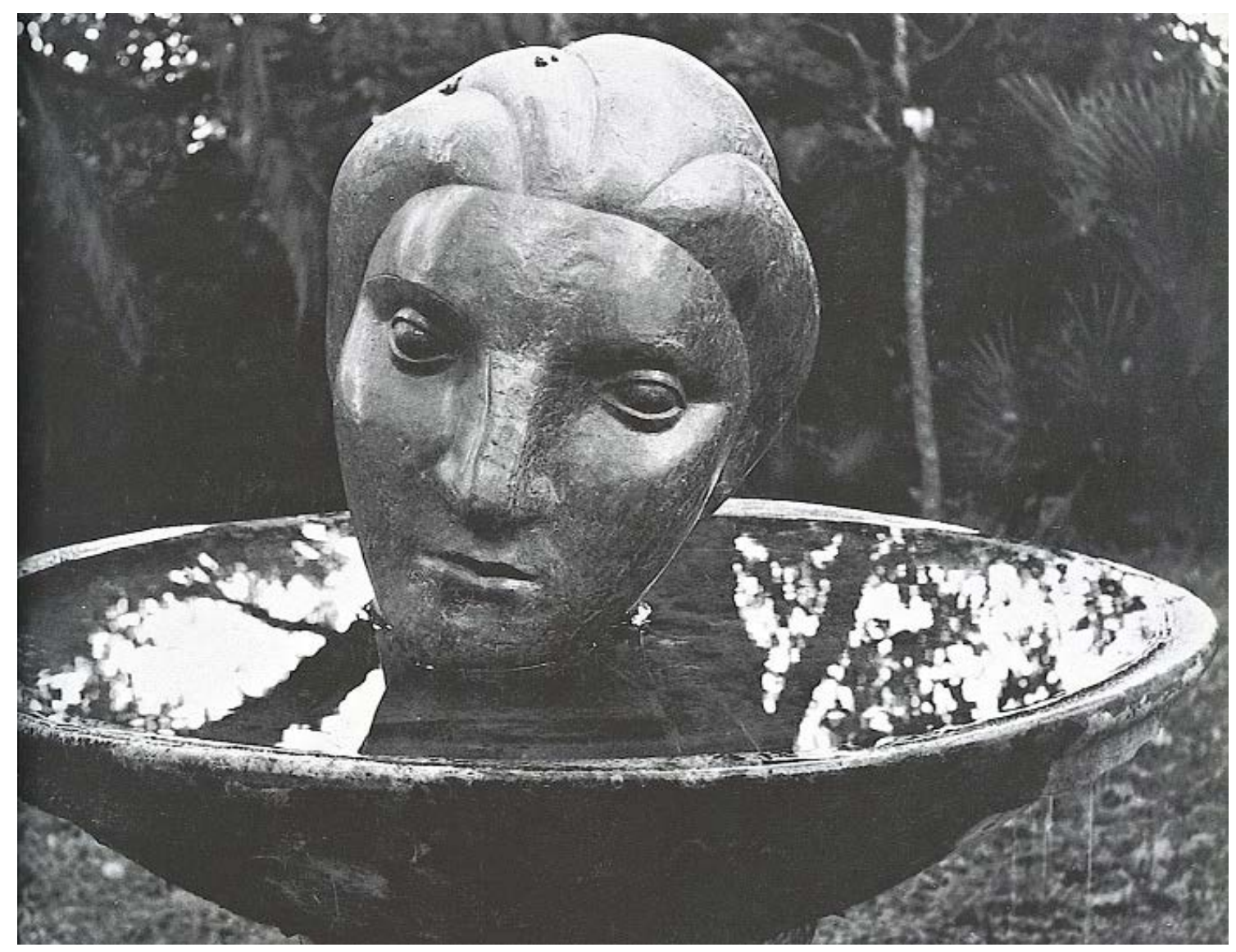

Lám. 2- Picasso, Cabeza de María Teresa Walter I, Boisgeloup, 1931.

(Ann M, Caws, Dora Maar con y sin Picasso, Barcelona, Destino, 2000)

La colocación de la réplica en bronce sobre la fuente fue un montaje del propio artista, que ofreció una imagen inédita en la que se aprecia la intensidad naturalista, el equilibrio con la monumentalidad de las formas esenciales y las cualidades innatas sobre las que procedió en los siguientes estados de la serie. 


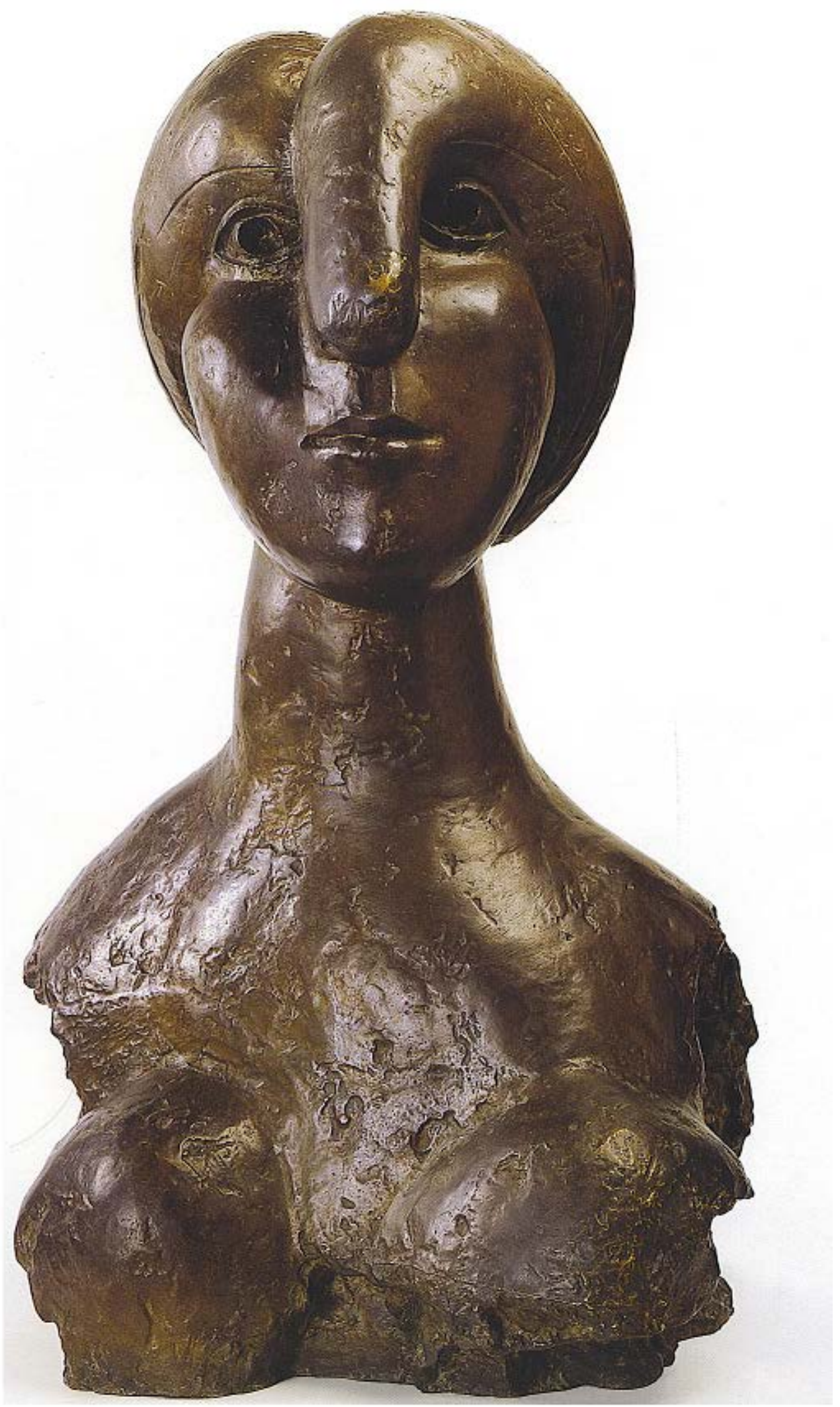

Lám. 3- Picasso, Busto de María Teresa Walter, Boisgeloup, 1931.

(Werner Spies, Picasso Sculpteur, Paris. Editions du Centre Pompidou, 2000)

La estructura nariz frente proporcionó la autonomía necesaria a los volúmenes en extensión superpuestos. 


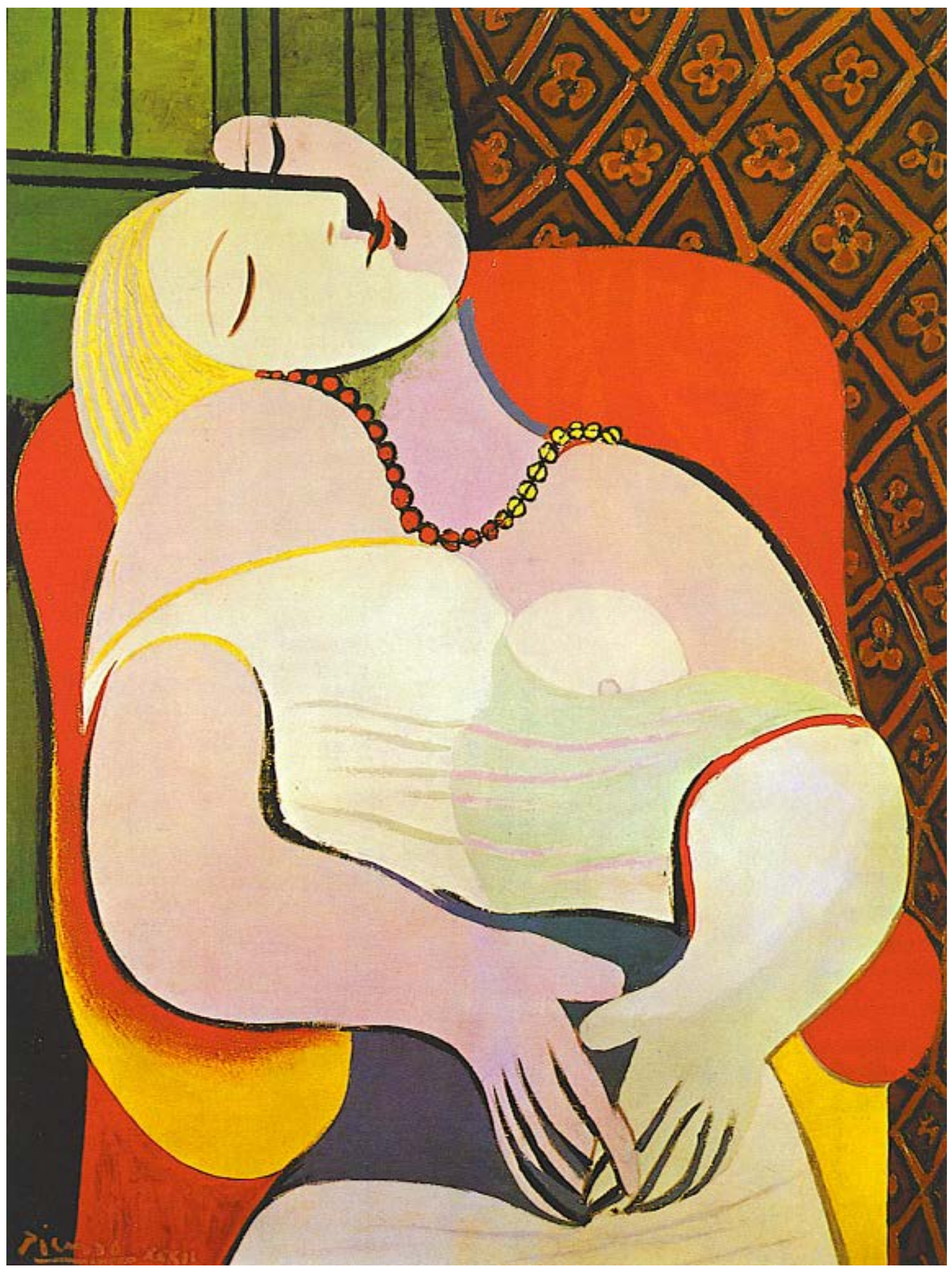

Lám. 4- Picasso, El sueño, Boisgeloup, 1932. Colección Mrs. Víctor W. Ganz.

(Warncke, Peter-Carsten, Pablo Picasso, 1881-1973. Köln, Taschen, 1997)

La estructura nariz frente adquirió un carácter estructural, válido en las proyecciones lineales en el plano y en los volúmenes reales. 


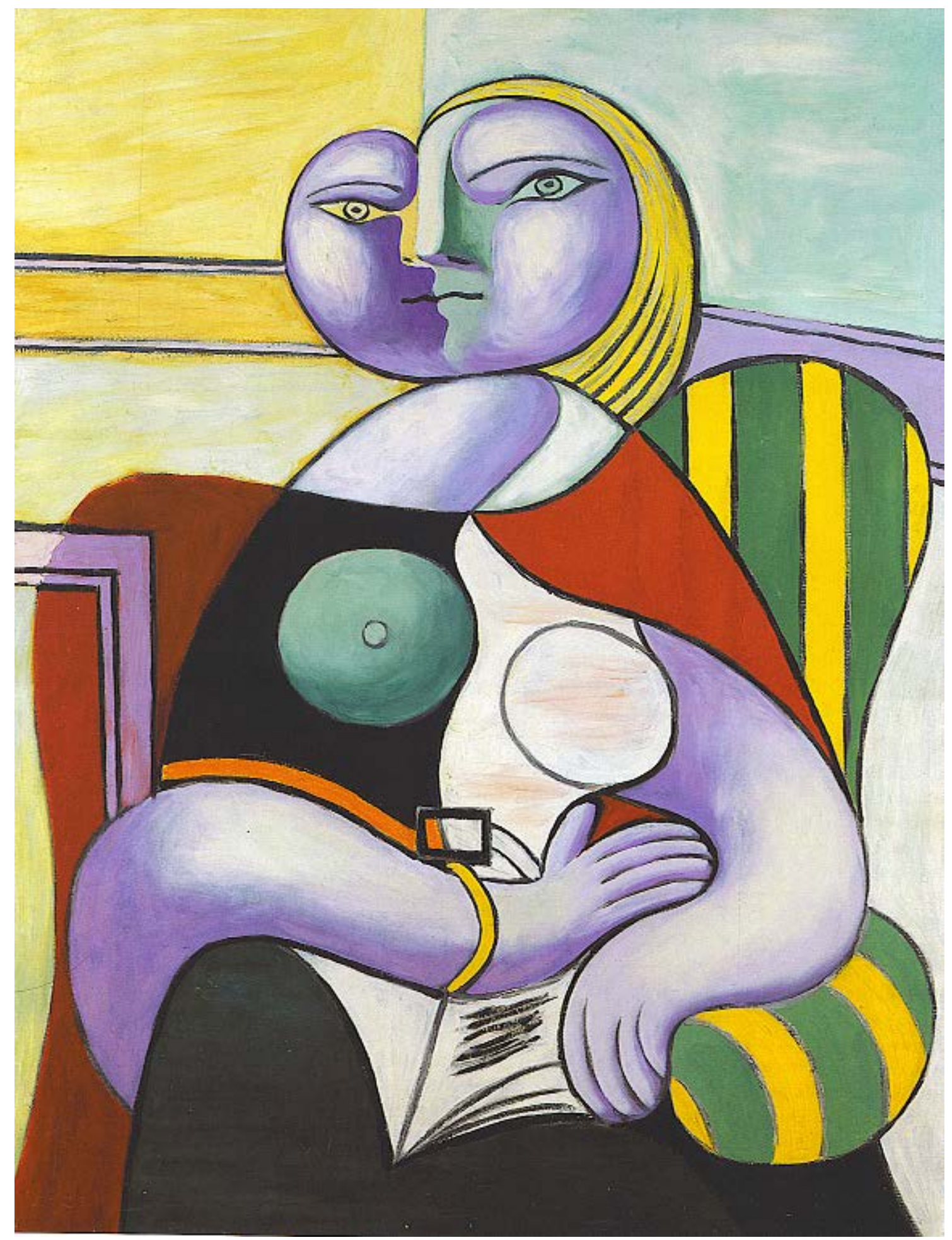

Lám. 5- Picasso, La lectura, Boisgeloup, 1932. Museo Picasso, París

(Warncke, Peter-Carsten, Pablo Picasso, 1881-1973. Köln, Taschen, 1997)

El carácter estructural de la nariz frente y sus posibilidades en el plano y la tercera dimensión posibilitaron las continuas inversiones de valores, éstas mediante trabajos escultóricos en positivo y negativo y pinturas lineales y tridimensionales. 


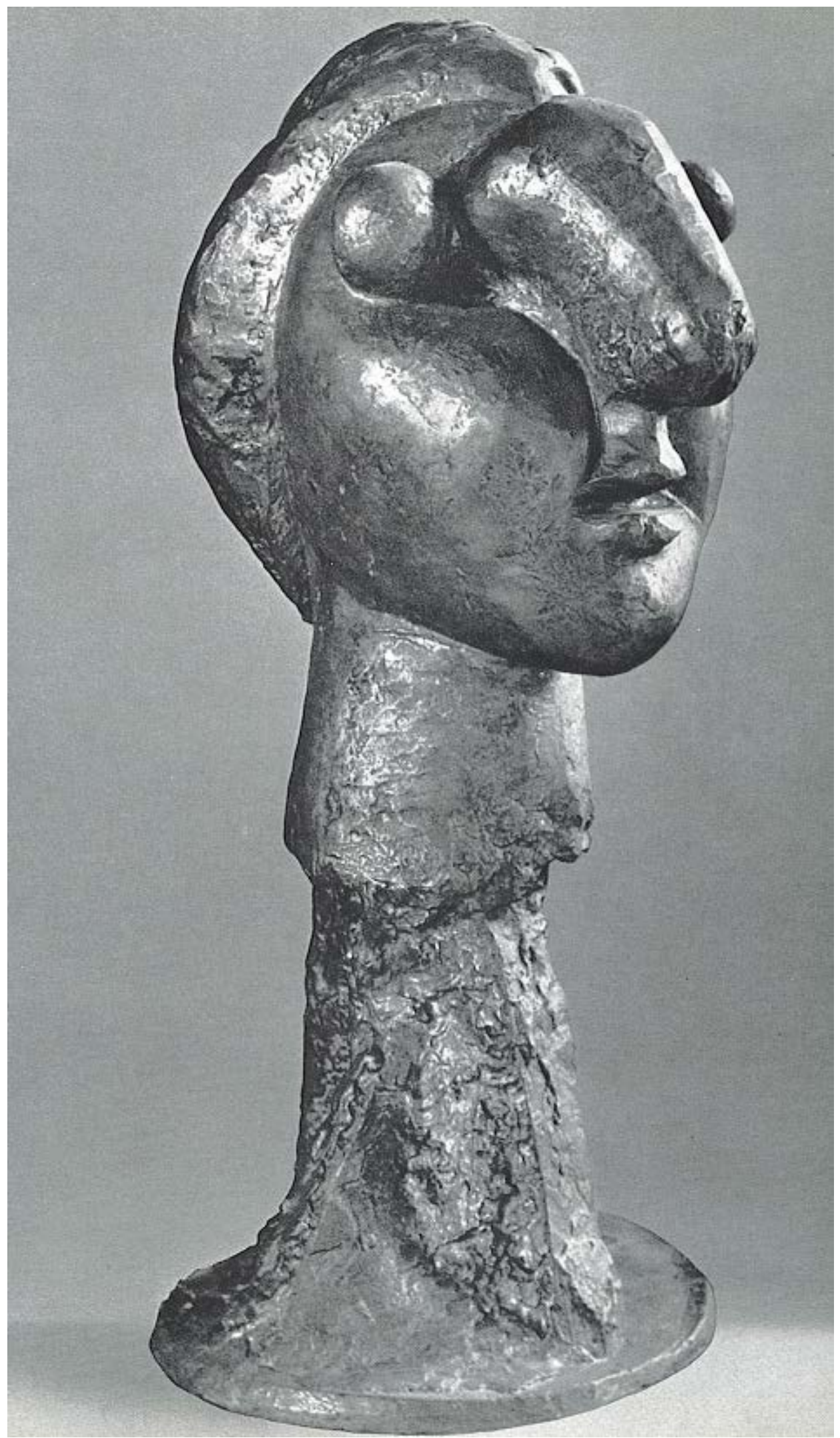

Lám. 6- Picasso, Cabeza de María Teresa Walter II, Boisgeloup, 1931.

(Brassï (Introduction Daniel-Henry Kahnweiler), Les Sculptures de Picasso. París, 1947)

Es la segunda escultura que Picasso vació en cemento para el Pabellón de España en la Exposición Internacional de París, en 1937. 


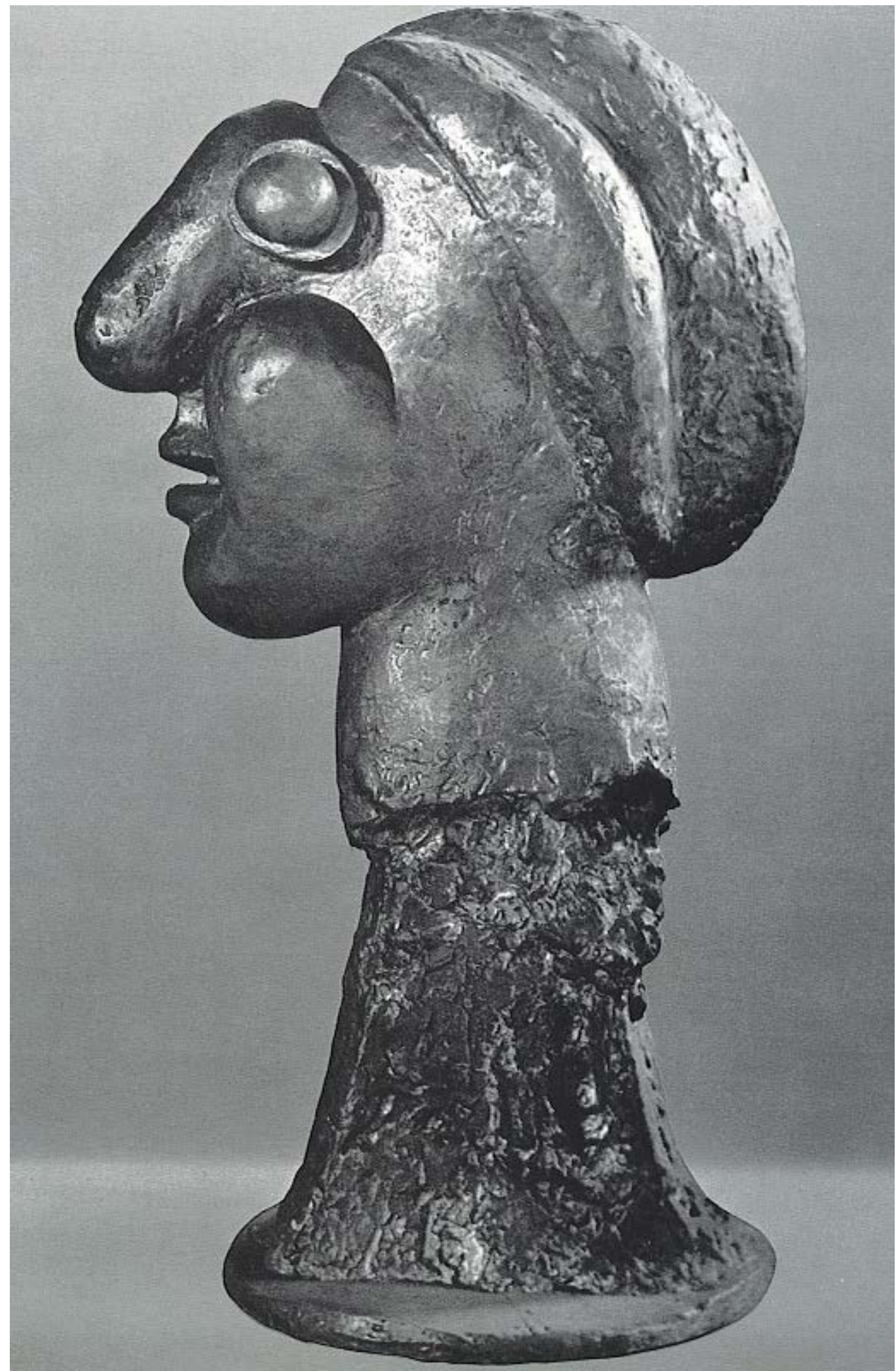

Lám. 7- Picasso, Cabeza de María Teresa Walter II, Boisgeloup, 1931.

(Brassï (Introduction Daniel-Henry Kahnweiler), Les Sculptures de Picasso. París, 1947)

Las cualidades originales del yeso fueron fundamentales para la proyección de los volúmenes en extensión y los contrastes de texturas que completan la configuración. 


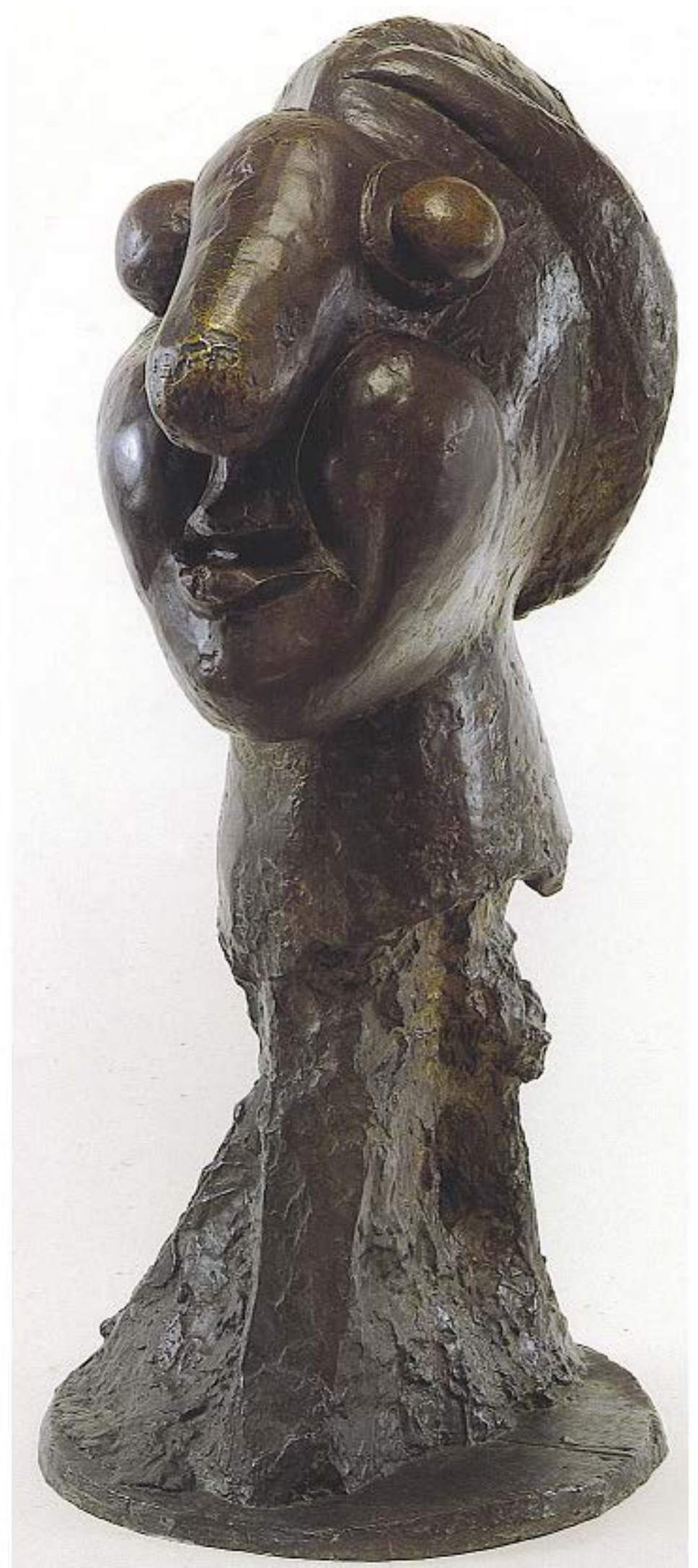

Lám. 8- Picasso, Cabeza de María Teresa Walter II, Boisgeloup, 1931.

(Werner Spies, Picasso Sculpteur. Paris. Editions du Centre Pompidou, 2000)

La réplica en bronce presenta una variante en el tratamiento rebajado de la base que sustenta al cuello. 


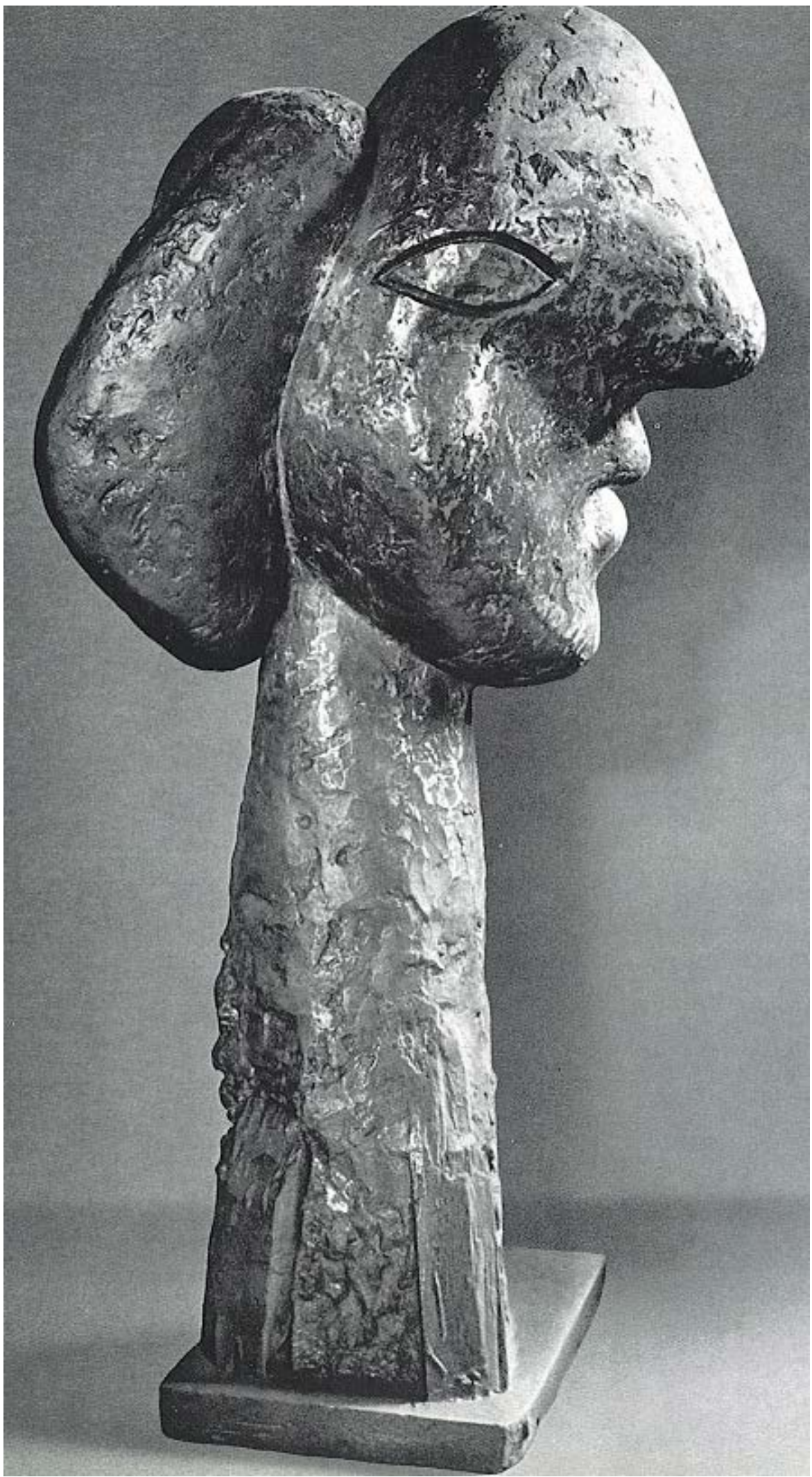

Lám. 9- Picasso, Cabeza de Maria Teresa Walter III, Boisgeloup, 1931.

(Brassï (Introduction Daniel-Henry Kahnweiler), Les Sculptures de Picasso. París, 1947)

Es la tercera escultura que Picasso vació en cemento para el Pabellón de España en la Exposición Internacional de París, en 1937. 


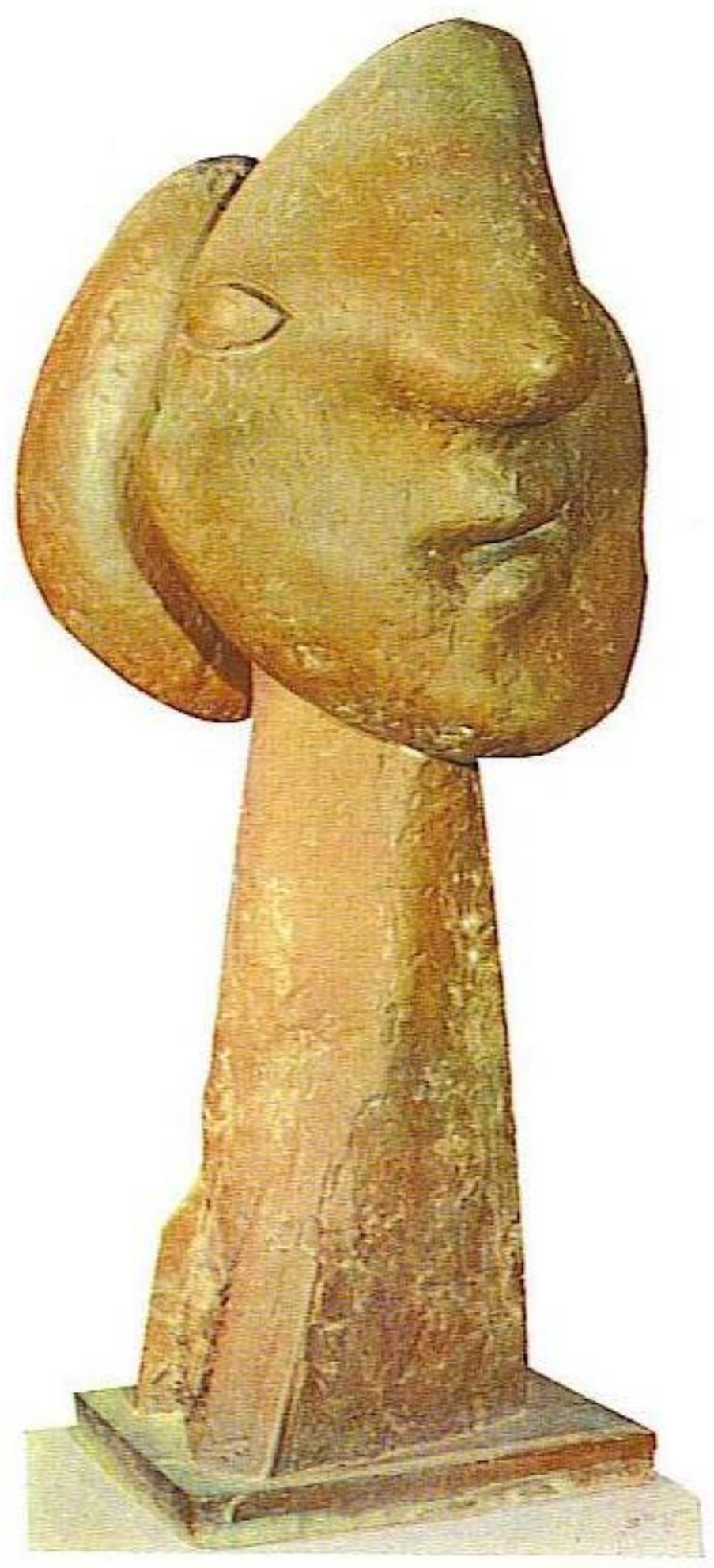

Lám. 10- Picasso, Cabeza de María Teresa Walter III, Boisgeloup, 1931.

El protagonismo de la nariz frente es proporcional a la radical simplificación de los volúmenes y las superficies y a la fuerza expresiva del modelado en extensión. 


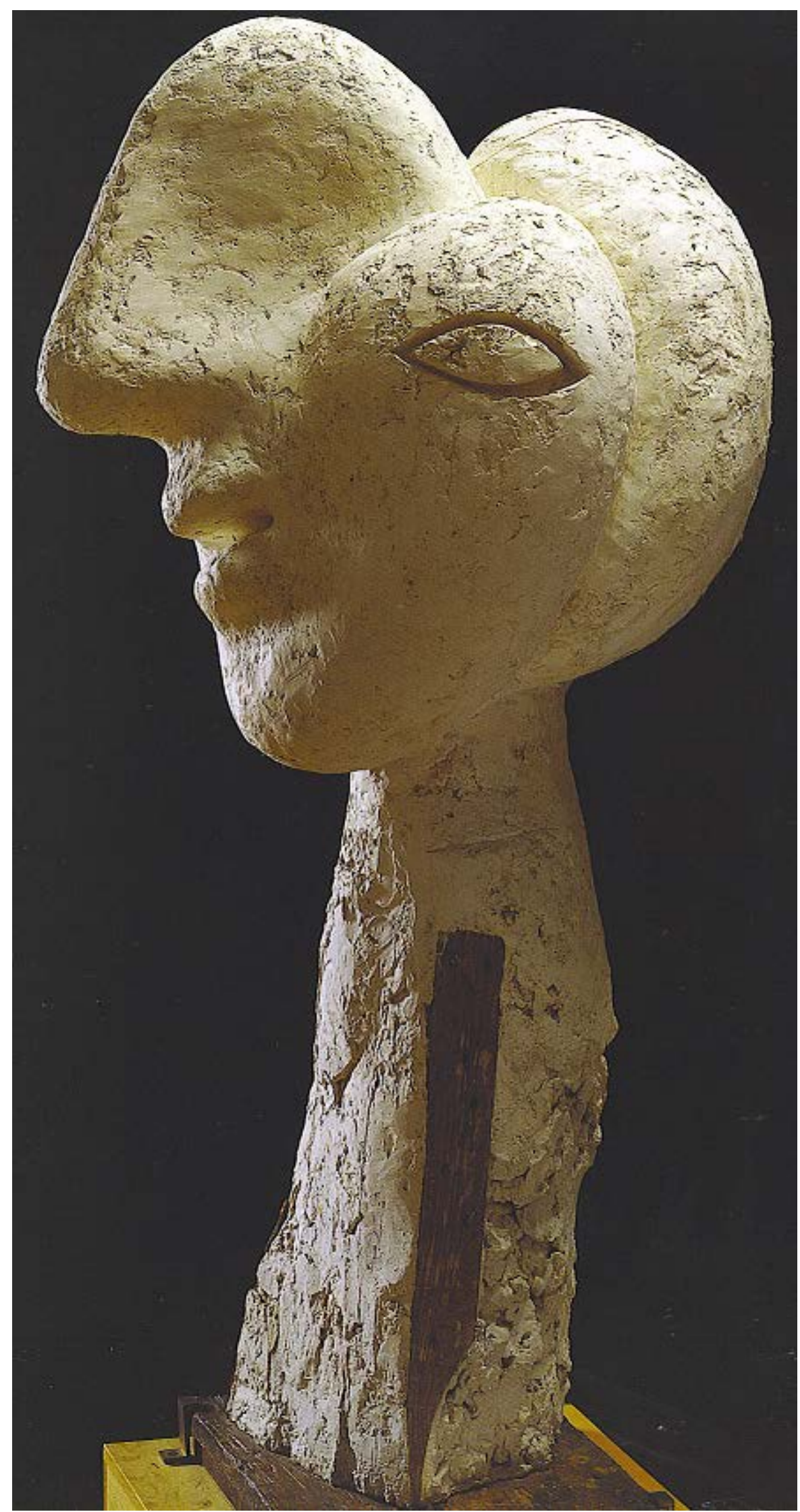

Lám. 11- Picasso, Cabeza de María Teresa Walter III, Boisgeloup, 1931.

(Spies, Werner, Picasso sculpteur. Paris. Editions du Centre Pompidou, 2000)

La monumentalidad de la composición significa sobre la radical simplificación formal. 


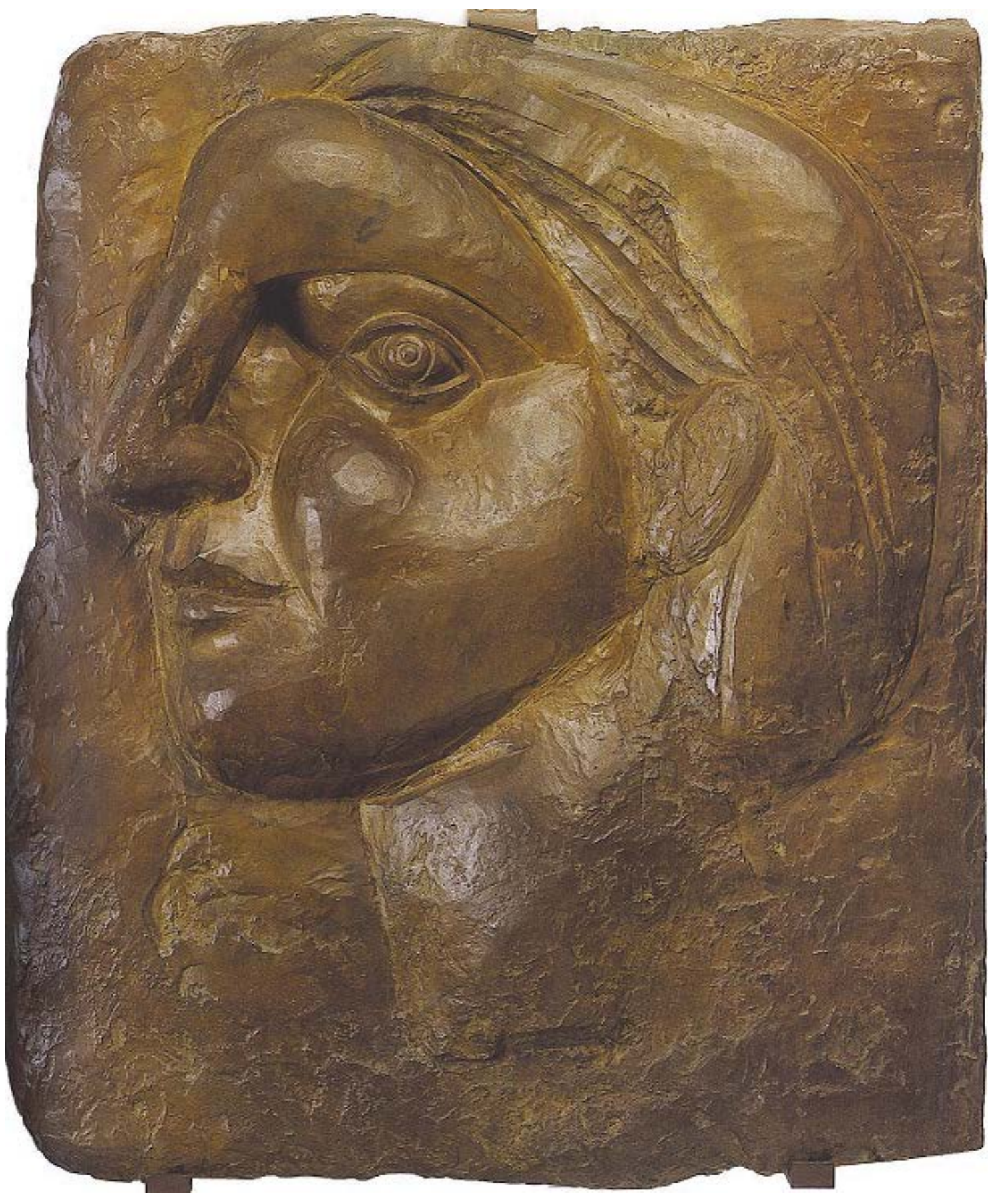

Lám. 12-Picasso, Cabeza en relieve de María Teresa Walter I, Boisgeloup, 1931.

(Spies, Werner, Picasso sculpteur. Paris. Editions du Centre Pompidou, 2000)

Las soluciones en el plano incentivaron la autonomía de los elementos naturales del retrato y la contundencia volumétrica es pareja a la intensidad expresiva. 


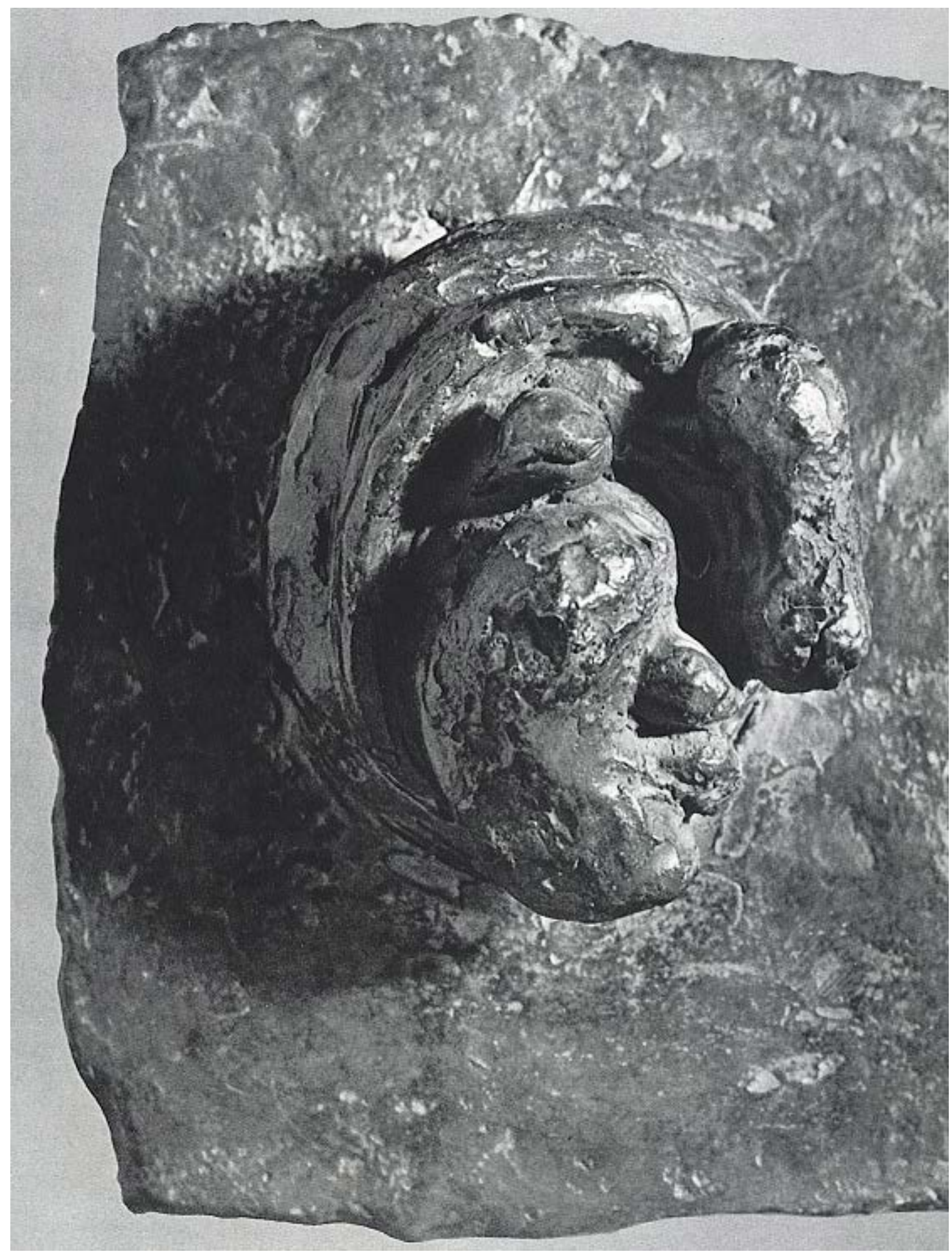

Lám. 13- Picasso, Cabęa en relieve de María Teresa Walter II, Boisgeloup, 1931.

(Brassï (Introduction Daniel-Henry Kahnweiler), Les Sculptures de Picasso. París, 1947.)

La progresiva autonomía de los volúmenes indica la evolución de la aplicación. 


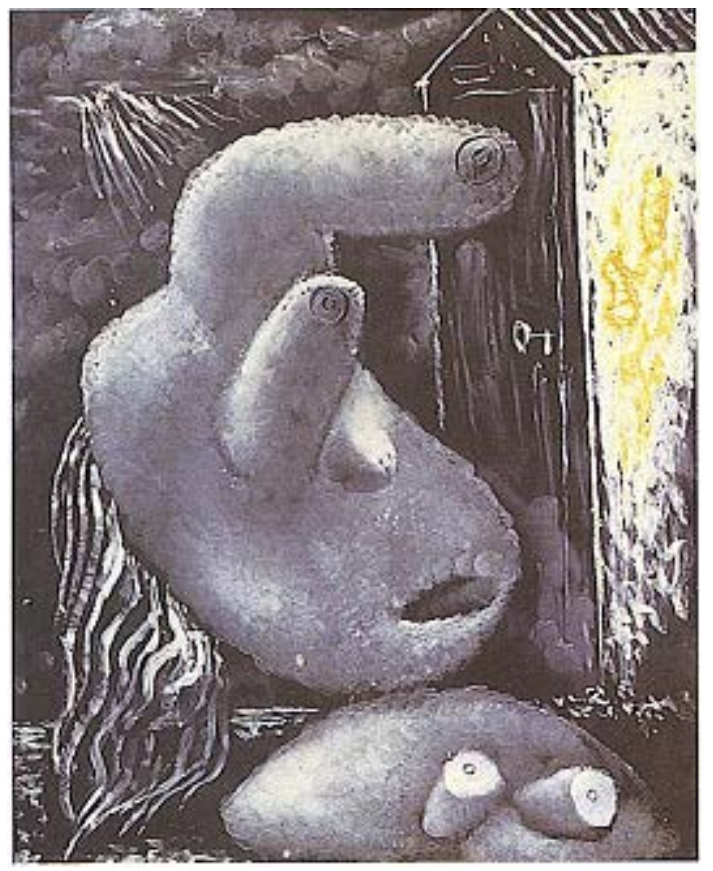

Lám. 14- Picasso y Dora Maar, Muchacha delante de una caseta de playa, Dinard, 1936-1937.

(Caws, Mary Anne, Dora Maar con y sin Picasso. Barcelona, Destino, 2000)

Óleo sobre placa de vidrio. Picasso mantuvo la vigencia de los conceptos escultóricos iniciados en los dibujos escultóricos de Cannes, en 1927, durante más de diez años y en cualquier género que resultase apropiado u oportuno.

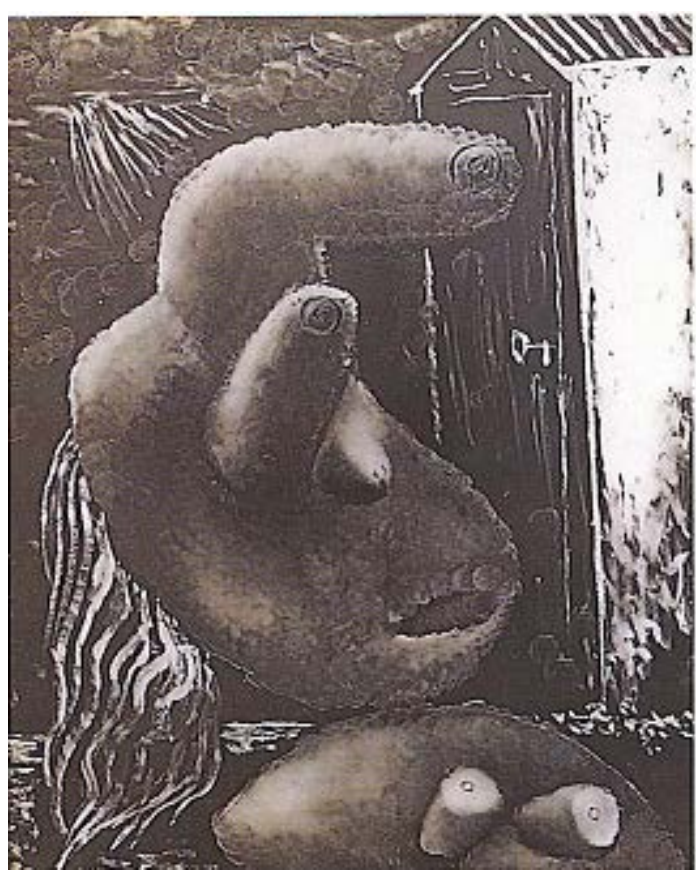

Lám.14- Picasso y Dora Maar, Muchacha delante de una caseta de playa, Dinard, 1936-1937.

(Caws, Mary Anne, Dora Maar con y sin Picasso. Barcelona, Destino, 2000)

Impresión de la imagen anterior sobre papel fotográfico. Las inversiones de valores y las continuas transformaciones formales contribuyeron a la proyección del medio fotográfico en las técnicas mixtas de carácter pictórico y la reproducción de réplicas intencionadas 